\title{
Performance analysis of dynamic spectrum leasing strategies in coordinated cognitive radio networks
}

\author{
Mario A. Ramírez-Reyna1* (D), Felipe A. Cruz-Pérez' ${ }^{1 *}$ S. Lirio Castellanos-López² , Genaro Hernandez-Valdez² \\ and Mario E. Rivero-Angeles ${ }^{3}$
}

\begin{abstract}
In this paper, dynamic and anticipated resource leasing strategies to address the problem of leased resource availability are investigated. Then, network performance analysis is developed, and the performance evaluations of different leasing strategies (namely permanent, dynamic, and anticipated leasing strategies) are provided for some network scenarios. The developed mathematical model additionally considers non-homogeneous bandwidth requirement, where different primary users (PUs) and secondary users (SUs) may require a number of channels. The tradeoff between spectrum leasing cost and system Erlang capacity (defined as the maximum offered traffic for which all the QoS requirements are guaranteed) is also investigated. Specifically, the maximum Erlang capacity is obtained by optimizing the number of reserved channels for different traffic scenarios considering a maximum allowed number of simultaneously rented channels. Also, the minimum achievable cost per Erlang is obtained by considering a fractional number of leased channels. Additionally, the cost per Erlang of capacity as function of both the utilization factor of the primary resources and the maximum allowed number of simultaneously rented channels is evaluated. Numerical results demonstrate that system performance strongly depends on the value of the mean secondary service time relative to the mean primary service time. Also, numerical results show that the cost of leasing a spectrum band is drastically reduced (compared to the permanent leasing strategy) when dynamic spectrum leasing strategies are employed.
\end{abstract}

Keywords: Coordinated cognitive radio networks, Dynamic spectrum leasing, Erlang capacity, Call admission control, New call blocking and call forced termination probabilities

\section{Introduction}

Recently, interest for cognitive radio networks (CRNs) to provide applications with stringent delay constraints has increased [1-5]. Nonetheless, providing this type of services is challenging due to the unpredictable nature of channel availability [3]. Several mechanisms have been proposed to reduce service interruption and/or to provide quality of service (QoS) provisioning in CRNs [3, 4, 6]. Among the most relevant of these mechanisms are spectrum handoff, call buffering, preemptive priority, channel reservation, spectrum adaptation, and spectrum leasing [4, 7-9]. Several research studies have shown that enabling leasing mechanisms allows to achieve a

\footnotetext{
* Correspondence: mramirezr@cinvestav.mx

${ }^{1}$ Electrical Engineering Department CINVESTAV-IPN, Mexico City, Mexico Full list of author information is available at the end of the article
}

better QoS related to the secondary users (SUs) in coordinated cognitive radio networks (CCRNs) [9-19]. A CCRN is formed by primary, secondary, and leasing sub-networks [5]. In the literature, the primary and leasing networks are fused in the same network [9]. The spectrum leasing mechanism means that the leasing sub-network rents to the secondary network part of its spectrum in exchange of some incentive (e.g., monetary rewards as leasing payments) [11]. The leased spectrum band could be purchased, or it could be leased as requested by the administrator of the secondary network. Under dynamic spectrum leasing regime, whenever the secondary network needs to rent more resources, it sends a request to the leasing network. The leasing network accepts or rejects such requests according to the availability of the resources in its network. To reduce 
the economic cost of renting resources through spectrum leasing, channels should be rented only when needed and should be released as soon as they are no longer needed. However, thanks to the random nature of channel occupancy, the resources to be leased cannot be guaranteed. To avoid this problem, in this paper, an anticipated leasing mechanism is proposed to increase the probability to find available channels to lease when needed.

In this work, we investigate the performance of dynamic spectrum leasing strategies (with and without anticipated resource leasing) for CCRNs with fixedrate traffic that has stringent delay constraints (such as the traffic flow generated by the unsolicited grant service class defined in Mobile WiMAX [20]). For comparison purposes, the permanent leasing strategy is also investigated. In the permanent spectrum leasing strategy, a fixed number of resources is permanently rented. On the other hand, the intuition behind the dynamic spectrum leasing strategy is to attempt to rent a channel only when needed and release it as soon as it is not needed. Finally, the intuition behind the anticipated spectrum leasing strategy is to attempt renting resources in advance (and release rented resources only when a preset minimum number of resources is achieved) to increase the probability of having available resource to be used when needed. In the next section, we briefly review the previously related work and point out the contribution of the present work.

\subsection{Related work}

The merit of this section is to summarize the previously published studies that have addressed the performance of resource management strategies in CRNs/CCRNs with stringent delay-sensitive traffic.

In relation to CRNs, let us describe the following related work. Authors in [21] analyzed the fractional guard channel reservation scheme to limit the forced termination probability of SUs in a CRN in which the spectrum handover technique is employed. In a related work [3], the optimal admission control and channel allocation decisions in cognitive overlay networks to support delay-sensitive communications of unlicensed users are derived. On the other hand, in [14], considering multiple classes of SUs, a heterogeneous-prioritized spectrum sharing policy for coordinated dynamic spectrum access networks is proposed. Additionally, authors in [14] develop two CAC strategies based on the channel reservation concept to enhance the maximum admitted traffic of SUs for the system. One of the CAC strategies proposed in [14] reserves channels for exclusive use of PUs (known as guard channels (GCs)), and the rest of channels (known as shared channels (SC)) are shared by all users (both PUs and SUs). In this strategy, a restriction function is used to balance the resource allocation between the different types of SUs. The other CAC strategy proposed in [14] considers two types of SUs; as such, the channels are divided into three groups: GCs, SCs, and restricted channels (RCs). RCs are reserved for the exclusive use of one type of SUs and cannot be used by the other type of SUs. Authors in [9] proposed a spectrum sharing scheme in which the dynamic spectrum access among PUs and SUs is coordinated by dividing the spectrum into normal access channels (that may be taken back anytime by PUs) and reserved secondary channels (that are locked by occupied SUs until call sessions are complete). Contrary to the work presented in $[3,9,14,21]$, in this paper, we consider spectrum leasing and channel reservation to prioritize ongoing secondary calls over new ones. Also, contrary to $[3,9,14,21]$, we consider a CCRN based on three sub-networks (primary, leasing, and secondary subnetworks).

Let us now describe the related work in the context of CCRNs. Authors in [18] investigated the performance of a CRN in which the primary network performs spectrum leasing considering the QoS requirements of both primary and secondary users. Additionally, in [18], a dynamic pricing scheme for spectrum leasing and utilitybased resource allocation is introduced, and the performance of dynamic leasing is compared against that of static leasing and approaches without leasing. Authors in [22] proposed a mechanism that incentivizes PUs to release resources for cooperation with SUs by means of a reward function. Other related work is [23], in which a CCRN model that comprises two primary networks and one secondary network is proposed. The two primary networks lease resources to a secondary network on different time intervals. To maximize the throughput of the three networks, a lease scheduler is implemented to guarantee the QoS needed for each network. In [24], a leasing mechanism based on resource reservation from a primary network is proposed and analyzed. Authors in [25] proposed a game theory solution for resource leasing through the usage of long-term contracts with PUs and short-term contracts with SUs for the distribution of the leased channels. Finally, in our early work [5], we studied the Erlang capacity ${ }^{1}$ in CCRNs with delay-sensitive traffic. However, in [5], the impact of the mean service time of SUs on system performance is not addressed. Opposite to [18, 22-25], in this paper, a third sub-network is considered as the one that leases the channels from the main CCRN and the system performance in terms of both spectrum leasing cost and system Erlang capacity is evaluated. This allows us to find relevant performance issues not previously reported in the 
existing literature. Moreover, in the present work, the maximum Erlang capacity is obtained and the factors that impact system Erlang capacity are investigated.

In our early work [26-28], we address the performance of spectrum leasing strategies in CCRNs. Similar to this paper, delay-sensitive secondary traffic is considered in [26, 28], while in [27], delay tolerant secondary traffic is considered. Contrary to [27], in this paper, we developed a teletraffic analysis for the performance evaluation of CCRN with delay-sensitive secondary traffic that considers non-homogenous use of radio resources by the primary and secondary users. Therefore, in [27], a different secondary traffic model is considered and a buffer for interrupted secondary calls is used. Thus, the developed teletraffic analysis in [27] is different to the one developed in this paper (i.e., one additional state variable is employed in [27]). Also, in [27], one additional different performance metric to the ones considered in this paper is evaluated (the mean delay transmission time). In addition, contrary to [28], in this paper, a CCRN based on three sub-networks (primary, leasing, and secondary sub-networks) is considered. Moreover, Castellanos-López et al. [28] only consider the permanent leasing strategy, while in this paper, dynamic spectrum leasing strategies are considered. Additionally, contrary to $[26,28]$, in this paper, two different theorems that describe the behavior of the CCRN in terms of the Erlang capacity are built. Contrary to $[26,27]$, the performance of dynamic, anticipated, and permanent spectrum leasing strategies is evaluated and compared under a more wide set of scenarios. Furthermore, contrary to [26], in this paper, the physical meaning of each equation along the paper is explained in detail.

The contribution of our present research work can be summarized as follows:

- A session-level teletraffic model for the performance evaluation of CCRNs with dynamic spectrum leasing for hard-delay-sensitive traffic is developed. This teletraffic model considers CCRNs based on three sub-networks (primary, leasing, and secondary sub-networks), where the leasing sub-network is considered as the one that lease the channels from the main CCRN. The developed teletraffic model includes, as special cases, our proposed (practical and ideal) anticipated spectrum leasing strategy and the permanent spectrum leasing strategy. Our developed mathematical framework considers nonhomogeneous bandwidth requirement (i.e., different number of channels utilized) for PUs, users of the leasing sub-network (RUs), and SUs. Also, a fractional reservation mechanism is implemented to prioritize ongoing secondary calls over new ones $[21,29]$; this is to set a fair comparison between the techniques.

- Building on our developed teletraffic model, general mathematical expressions for the new call blocking and forced termination probabilities, arrival rate of secondary users of the leasing network, and fraction of time during which the secondary network uses leased radio resources are derived.

- For a given QoS requirement at connection level (i.e., call blocking and forced termination probabilities) and considering a maximum allowable number of simultaneously rented channels, maximum Erlang capacity is obtained by optimizing the number of reserved channels for different traffic scenarios (i.e., scenarios with different values of the ratio between mean secondary service time and mean primary service time, and different values of the utilization factor of both primary and rented channels). Building on this, the factors that impact system Erlang capacity are investigated. To the best of the authors' knowledge, the impact of the relative value of the mean service time of SUs to the mean service time value of PUs on system performance has not been previously reported in the literature.

- The economic cost of spectrum leasing per Erlang of capacity is investigated. Also, the minimum cost of rented resources per Erlang of capacity is obtained by (mathematically) allowing the number of leased resources to be real (i.e., a fractional number of rented resources is considered).

- The performance of dynamic, anticipated, and permanent spectrum leasing strategies are evaluated and compared under different traffic conditions and system parameters in terms of maximum Erlang capacity, economical cost of spectrum leasing per Erlang of capacity, and optimal number of leased channels. This study has allowed us to find relevant insights into the performance of spectrum leasing strategies in CCRNs. For instance, numerical results show that the cost of leasing a spectrum band is drastically reduced (compared against the permanent leasing strategy) when dynamic spectrum leasing strategies are employed. Also, numerical results reveal that system performance strongly depends on both the mean secondary service time relative to the mean primary service time and the value of bandwidth requirement of both primary and secondary users.

The rest of this paper is organized as follows. System model and general assumptions are given in Section 2.1. Also, in Section 2.1, the different studied leasing strategies (i.e., permanent, dynamic, practical anticipated, 
and ideal anticipated leasing strategies) and the used admission control strategy are described. Our proposed mathematical teletraffic model for the performance analysis of dynamic spectrum leasing strategies in CCRNs with delay-sensitive traffic and fractional channel reservation is developed in Section 2.2. Also, in Section 2.2, mathematical expressions for the different system performance metrics are obtained. Numerical results are analyzed in Section 3, before concluding remarks are exposed in Section 4.

\section{Method}

\subsection{System model}

In this section, the main assumptions and system parameters are explained in detail. The mathematical model developed in this work is based on [9]; this model closely reflects a CCRN with spectrum leasing capabilities for delay-sensitive traffic. In contrast with [9], a third sub-network that leases channels to the secondary sub-network is considered (this serves as a priority scheme to guarantee the QoS of the SUs). From the teletraffic analysis point of view, the main difference of this work and that in [9] is the utilization of an additional state variable to represent the number of users with ongoing sessions in the referred third (leased) sub-network. Thus, the CCRN is formed by three wireless sub-networks working in a given service area. The wireless sub-networks that have the rights to operate on the given bands are referred to as the primary (PS) and leasing (RS) sub-networks (as it is illustrated in Fig. 1). The other sub-network in the same service area is referred to as the secondary sub-network (SS), which opportunistically shares the spectrum resources with the PS (while causing negligible interference to PUs of the PS) and dynamically interacts with the RS leasing radio resources. (Traditionally, PS plays also the role of the RS [3].) Naturally, the RS and SS sub-networks have to be coordinated; consequently, the CCRN requires each of these two sub-networks to exchange data about the state of each network. However, the reduction in the interaction

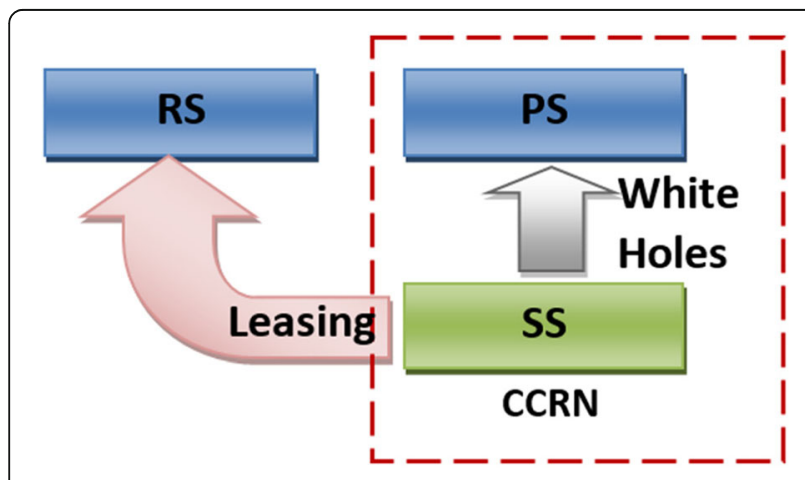

Fig. 1 Architecture of the three-sub-network CCRN between RS and SS is desirable to minimize the awareness that the RS needs to have of the SS activities [10]; in this work, the information that the RS shares with the SS is just about the actual state of occupancy. Primary users of the RS sub-network (RUs) compete for the channel usage against new SUs and SUs in spectrum handoff whose sessions cannot be carried by the PS due to the lack of available channel resources. Hence, SUs may interpose with the performance of the RUs. On the other hand, primary users (PUs) do not need to know about SU's activities, and SUs already detect activities of PUs through spectrum sensing. Hence, SUs are transparent to PUs.

Referring to spectrum sensing, it has been shown in the literature $[4,30-33]$ that the effect of false alarm and misdetection can be easily plugged into most of the developed mathematical analysis of CRNs. However, in this paper, to keep our mathematical analysis simple, we assume ideal spectrum sensing (i.e., spectrum sensing is considered error-free). This represents a reasonable assumption in CRNs where a sensor network having enough sensors performing collaborative sensing of the radio environment in space and time is employed as in $[3,11]$. Modeling of spectrum sensing error in CRNs has been addressed in $[4,30-33]$. (For the interested reader, the effect of unreliable spectrum sensing in CRNs under voice over Internet Protocol traffic is investigated in our early work [4].) Also, it is considered that the probability that any service event happening during the sensing period is relatively small.

In this paper, the system performance of the downlink of a wireless CCRN is considered [34]. ${ }^{2}$ Also, we consider a single base station (i.e., evolved node B (eNB) in LTE vocabulary) that attends transmissions of SU among $N$ primary channels with up to $R$ allowable channels to be leased. For simplification, along the paper, we refer to a channel as a resource block in a LTE system. Also, a fair channel model is considered, where all channels have the same data rate. As in [21], because blocking of ongoing secondary calls is more problematic than blocking of new SU call arrivals, a resource reservation is considered; this reservation consists in a fractional number $r$ of channels (composed of rented and/or normal sub-channels). Considering $r$ as a real number and $0 \leq$ $r \leq N$, each normal or rented channel can handle only one secondary session. Thus, the system can handle up to $N+R-r$ simultaneous secondary sessions. In order to have a more accurate and fair comparison for the Erlang capacity in terms of the operational cost, real values for the number of leased and reserved channels are considered. Indeed, in order to find the exact value of the Erlang capacity among all the considered strategies, real values for $r$ and $R$ should be considered. Otherwise, the small differences among the fractional values of the 
leased and reserved channels may cause a higher or lower operational cost of the system. Note that this is a mathematical tool and it is not intended for a practical implementation. On the other hand, for the RS sub-network, $K$ channels are assigned. For the PUs, there are $N$ channels that are shared with the SUs; it is important to note that the PUs have the priority over these channels. About the leased resources, only the SUs can access them. Figures 2 and 3 illustrate the resource distribution for the sharing models for each of the strategies (permanent and dynamical/anticipated strategies, respectively). It is important to mention that if SUs cannot find available resources when arriving or when are being interrupted, it can be redirected to the RS sub-network by using any of the proposed leasing mechanisms. For the sake of the analysis, it is considered that SUs requesting service are able to connect and stay connected up to the end of their sessions (if there are enough available resources to this end) to this base station, that is, no handoff mechanism is considered (typical in low mobility scenarios).

The number of RUs, PUs, and SUs at any time is represented by state variables $l, m$, and $n$, respectively. The arrival rates of the PU, SU, and RU are independent Poisson processes with arrival rates $\lambda_{l}, \lambda_{m}$, and $\lambda_{n}$, respectively. Service times for PU, SU, and RU are independent exponentially distributed random variables with mean values $1 / \mu_{1}, 1 / \mu_{\mathrm{m}}$ and $1 / \mu_{\mathrm{n}}$, respectively. The number of channels required by sessions of RUs, PUs, and SUs is $b_{l}, b_{m}$, and $b_{n}$, respectively. In Table 1 , a list of the variables and acronyms used along the paper is provided.

\subsubsection{Coordinated cognitive radio network model}

As mentioned before, there are two types of CCRN, a CCRN with two sub-networks and a CCRN with three sub-networks. In a CCRN with two sub-networks, the cognitive radio leases the resources to the same system that provides the $\mathrm{CR}$ capabilities (i.e., the same primary

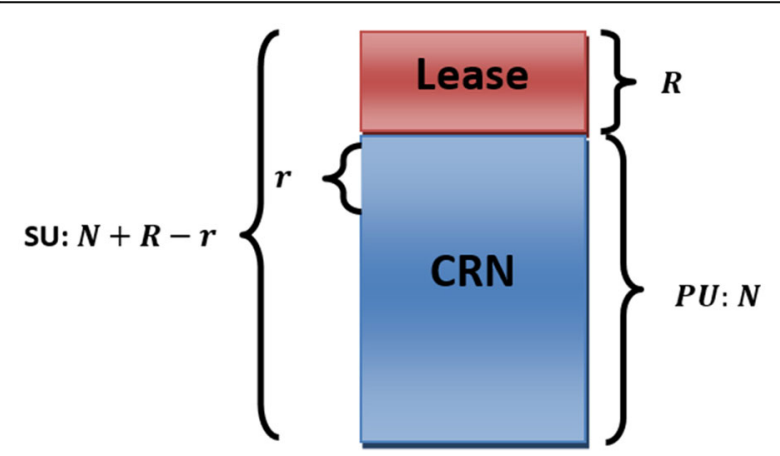

Fig. 2 Resource distribution and allocation for the permanent leasing strategy

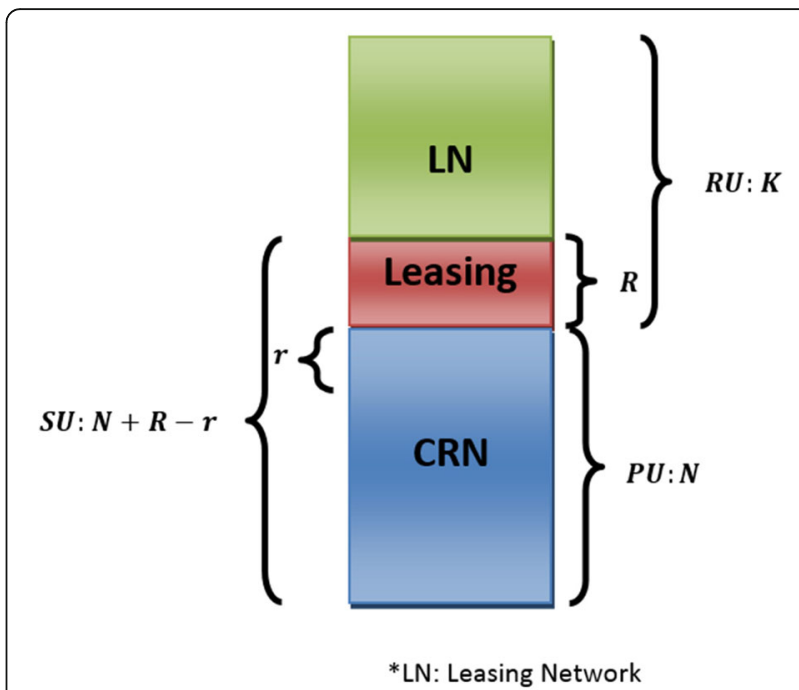

Fig. 3 Resource distribution and allocation for the dynamical and anticipated leasing strategy

sub-network is the one that lends the white spaces for the SU usage and, also, leases the channels when requested). In a CCRN with three sub-networks, SUs lease available channels from a third party (i.e., the RS sub-network). It is observed that the three sub-networks approach attains a better performance than the two sub-network one. This result is obvious thanks to the availability of the resources, meaning there are more available resources for the SUs to lease and exploit the CR capabilities. As such, the following leasing strategies are focused on this three sub-networks CCRN scenario. In this work, the following spectrum leasing strategies are studied and analyzed for a CCRN composed of three sub-networks. These spectrum leasing strategies perform as follows:

a) Permanent leasing strategy (PLS). The main idea behind this strategy is to permanently rent a fixed number of resources. In this strategy, the SS leases $R$ channels permanently to be used exclusively by the SUs from the RS (Fig. 2). This strategy is the simplest of the three and does not require interaction among the sub-networks. However, the main disadvantage of this strategy is the sub-utilization of the channels permanently leased because of a small probability that the SUs use those resources. As such, the cost of implementation/operation is high.

b) Dynamical leasing strategy (DLS). If a SU finds all the channels occupied in the PS, the SS dynamically leases a resource from the RS. The intuition behind this strategy is to attempt to rent a channel only when needed and release it as soon as it is no longer required. Note that the SS is able to lease up 
Table 1 List of variables and acronyms

\begin{tabular}{|c|c|}
\hline $\begin{array}{l}\text { Variable/ } \\
\text { acronym }\end{array}$ & Description \\
\hline$\Delta a_{\mathrm{RU}}$ & Increment in the offered traffic load of the RUs. \\
\hline$\Delta R_{\max }$ & Increment in the maximum number of leasing channels. \\
\hline$\lambda_{l}$ & Arrival rate of primary users to the leasing network. \\
\hline$\lambda_{m}$ & Arrival rate of primary users to the primary network. \\
\hline$\lambda_{n}$ & Global arrival rate of secondary users. \\
\hline$\lambda_{R}$ & Arrival rate of secondary users to the leasing network. \\
\hline$\mu_{l}$ & $\begin{array}{l}\text { Inverse of the mean service time for primary users of } \\
\text { the leasing sub-network. }\end{array}$ \\
\hline$\mu_{m}$ & $\begin{array}{l}\text { Inverse of the mean service time for primary users of } \\
\text { the primary sub-network. }\end{array}$ \\
\hline$\mu_{n}$ & Inverse of the mean service time for secondary users. \\
\hline$\rho_{P U}$ & Utilization factor for the primary channels. \\
\hline$\rho_{R U}$ & Utilization factor for the channels of the leasing network. \\
\hline$\Omega$ & Set of feasible states for the system. \\
\hline$a_{1}$ & Transition rate for user of the leasing network. \\
\hline$a_{m 1}$ & $\begin{array}{l}\text { Transition rate for primary users without forcing to } \\
\text { terminate any secondary user call. }\end{array}$ \\
\hline$a_{m 2}$ & $\begin{array}{l}\text { Transition rate for primary users with at least one } \\
\text { secondary user call terminated. }\end{array}$ \\
\hline$a_{n}$ & Transition rate for secondary users. \\
\hline ALS & Anticipated leasing strategy. \\
\hline$a_{s u}^{(o p t)}$ & Optimal offered traffic load of the SUs \\
\hline$a_{S U}{ }^{(n e w)}$ & Recalculated offered traffic load of the SUs \\
\hline$a_{\mathrm{RU}}$ & Offered traffic load of the RUs \\
\hline$b$ & Vector of value $\boldsymbol{b}=\left\{b_{m}, b_{n}\right\}$ \\
\hline$b_{1}$ & Resource requirement of users of the leasing network. \\
\hline$b_{m}$ & Resource requirement of user the primary users. \\
\hline$b_{n}$ & Resource requirement of secondary users. \\
\hline CAC & Call admission control \\
\hline CCRN & Coordinated cognitive radio network. \\
\hline$C R$ & Cognitive radio \\
\hline CRN & Cognitive radio network. \\
\hline$d_{1}$ & Departure rate for users of the leasing network. \\
\hline$d_{m}$ & Departure rate for primary users. \\
\hline$d_{n}$ & Departure rate for secondary users. \\
\hline DLS & Dynamic leasing strategy \\
\hline$e$ & Vector of value $\boldsymbol{e}=\{m, n\}$ \\
\hline$E\{L\}$ & Average number of channels leased. \\
\hline$E\{W \mid W>0\}$ & $\begin{array}{l}\text { Conditional average time that secondary users lease } \\
\text { resources. }\end{array}$ \\
\hline K & Total number of channels on the leasing network. \\
\hline 1 & $\begin{array}{l}\text { Number of users with ongoing sessions in the leasing } \\
\text { network. }\end{array}$ \\
\hline$m$ & $\begin{array}{l}\text { Number of primary users with ongoing sessions in the } \\
\text { system. }\end{array}$ \\
\hline N & Total number of channels in the CCRN. \\
\hline
\end{tabular}

Table 1 List of variables and acronyms (Continued)

\begin{tabular}{|c|c|}
\hline $\begin{array}{l}\text { Variable/ } \\
\text { acronym }\end{array}$ & Description \\
\hline$n$ & $\begin{array}{l}\text { Number of secondary users with ongoing sessions in } \\
\text { the system. }\end{array}$ \\
\hline$P(l, m, n)$ & Probability of being in the state $(1, m, n)$. \\
\hline$P_{r}$ & $\begin{array}{l}\text { Probability of blocking a new secondary user when } r+1 \\
\text { channels are reserved. }\end{array}$ \\
\hline$P_{R}$ & $\begin{array}{l}\text { Probability of admitting a new primary user when a } \\
\text { number } R+1 \text { of channels are permitted to be leased. }\end{array}$ \\
\hline$P_{\mathrm{bl}}$ & $\begin{array}{l}\text { Blocking probability for users from the leasing sub- } \\
\text { network. }\end{array}$ \\
\hline$P_{\mathrm{bm}}$ & Blocking probability for primary users. \\
\hline$P_{\mathrm{bn}}$ & Blocking probability for secondary users. \\
\hline PLS & Permanent leasing strategy. \\
\hline PS & Primary sub-network \\
\hline$P_{\mathrm{ftn}}$ & Forced termination probability for secondary users. \\
\hline PU & Primary User of the primary sub-network. \\
\hline Qos & Quality of service. \\
\hline$R$ & Number of channels allowed to be leased. \\
\hline$r$ & Number of reserved channels. \\
\hline$r^{(\mathrm{opt})}$ & Optimal number of reserved channels \\
\hline$r^{(\text {new })}$ & Recalculated number of reserved channels \\
\hline$R_{\max }$ & $\begin{array}{l}\text { Maximum number of channel allowed to be leased for } \\
\text { dynamic anticipated leasing. }\end{array}$ \\
\hline RS & Leasing sub-network. \\
\hline $\mathrm{RU}$ & Primary users of the leasing sub-network. \\
\hline SS & Secondary sub-network. \\
\hline SU & Secondary user \\
\hline$S_{S U}$ & $\begin{array}{l}\text { Number of resources being rented by the secondary } \\
\text { sub-network. }\end{array}$ \\
\hline
\end{tabular}

to $R$ channels (Fig. 3). Contrary to the PLS strategy, this strategy require a certain amount of awareness between the SS and the RS sub-networks. The main advantage of this method is that leased channels are always requested when needed; thus, there is no wastage or underutilization of resources. This translates in a cost-friendly system. Contrarily, the main disadvantage of this strategy is that there is no guarantee of the resource availability due to the RUs usage in the RS. It is important to note that it is possible that SUs fail to find idle channels when requesting for leasing resources even if the maximum allowable number of leased channels are not being used. To mitigate the impairment of this strategy, a third one was designed to reduce the dropped SU calls due to the arrival of PUs.

c) Anticipated leasing strategy (ALS). The intuition behind this strategy is to attempt renting resources in advance (and release rented resources only when 
a preset minimum number of resources is achieved) to increase the probability of having available resource to be used when needed. In this strategy, a channel is leased in advanced when certain events are detected (i.e., whenever a SU or a PU arrives to the PS). The rationale behind this strategy consists in the reduction of the interruption of $\mathrm{SU}$ sessions through the enabling of resources that have been leased in advance, thus mitigating the high interruption rates when the PS is in high occupancy. In this way, this strategy seeks to prevent the full usage of resources by the RUs by renting a channel in advance for each arrival event and thus increase the probability of finding available resources when requests are made by the SUs in the RS sub-network. It can be seen that the extra leased channel is not used immediately. Hence, this strategy is subject to resource wastage. The aim of this mechanism is to reduce the dropping probability of a SU call caused by the arrival of a PU. This dynamic spectrum leasing strategy is called practical anticipated spectrum leasing strategy. With respect to this strategy, we are interested in the minimization of the resource wastage (due to the anticipated lease of channels). In this sense, we consider that the minimum resource wastage occurs when a RU of the RS sub-network is about to use a channel and, an instant before this happens, the SS rents a channel. In a real system, it is not possible to achieve the minimum resource wastage because it cannot be predicted when a RU will make use of the resource in the RS sub-network. This limit case is called ideal anticipated leasing approach and is evaluated only for comparison purposes.

\subsubsection{Call admission control strategy}

In this work, a call admission control strategy where, from the SS sub-network perspective, a real number of $r$ channels of the PS are reserved for exclusive use of interrupted secondary calls. As such, a new secondary arrival is accepted if the following conditions are met:

a) With probability 1 when $b_{m} m+b_{n} n<=N+R-\lfloor r\rfloor$ $-b_{n}$, i.e., there exist enough available resources in the PS to accept the new secondary call request.

b) On the other hand, when there are $N+R-\lfloor r\rfloor-1$ $-b_{n}$ users in the CCRN and a new SU arrives, it is blocked with probability $P_{r}=r-\lfloor r\rfloor$ and it is accepted with probability $\left(1-P_{r}\right)$. The symbol “【]" denotes the floor operation.
On the other hand, a SU uses a leased channel in the following cases:

a) A PU arrives to the PS when all the $N$ channels are occupied (and at least one of these channels is occupied by SUs). In this scenario, all the secondary calls using one of these channels are interrupted to make room for the just arriving primary call request. All the interrupted secondary calls look for available rented resources at the RS sub-network. Obviously, available resources are needed at the RS sub-network; otherwise, all (or some) of the interrupted secondary calls are forced to terminate.

b) A new SU call arrives and all of the $N$ channels of the PS are in use. In this case, the new secondary call is transferred to the RS subnetwork if there are available channels; otherwise, it is blocked.

At any time, PUs have priority to acquire the channels of the PS that are used by SUs. A PU request is accepted if $b_{m} m \leq N-b_{m}$, meaning that there are enough resources to course a PU session; otherwise, it is rejected. When a PU is accepted, and the channels assigned to it are being occupied by calls of SUs, those calls must be relinquished. Every SU call that was preempted is transferred to an idle channel, if available, to resume its transmission. If there are no idle channels in the PS, preempted secondary calls are transferred to the RS and are assigned to idle leased channels. If there are no enough leased channels available to continue the service of the preempted secondary calls, then, part of these calls are forced to terminate.

For the purpose of reducing the resource wastage, if an available channel is detected in the PS and currently there are SU calls being carried on leased channels, one of those calls is transferred to the idle normal channel in order to reduce the occupancy of leased channels. These mechanisms are important when the dynamic spectrum leasing policy is seeking to reduce the cost of leasing a spectrum band.

In order to further illustrate the proposed anticipated leasing strategies, some operational examples are studied next.

In the first part of Fig. 4, we show a timeline of events in the anticipated leasing strategy for the practical approach. It can be seen that the system conditions are such that there are no available resources in the PS. Hence, when a PU arrives, a SU has to perform a spectrum handoff and searches for channels in the RS sub-network. According to the anticipated strategy, the incoming SU rents a channel and rents also an additional channel in order to guarantee 
available resources for other SUs arriving in these conditions. As such, at this point, the red line (that indicates the number of rented channels) climbs two steps, but only one of these rented channels is being used (observe the green area that represents the number of rented channels actually being used). Then, when another SU arrives to the system, it uses the previously rented channel and rents in advance another channel. Then, a PU arrives causing another spectrum handoff of a SU. The SU uses the previously rented channel, but the number of rented channels reaches its maximum value $R$. Hence, beyond this point, it is not possible to rent another channel in advance. After this point, a SU terminates its call. However, there remains an anticipated rented channel. This can also be seen as a delayed release mechanism. After some departures of PUs and SUs, a new SU arrives and it rents another channel in order to anticipate the arrival of another SU. Next, a session of a RU in the RS arrives. It uses one of the available channels, but this also triggers the anticipated rent of another channel. As such, when another RU arrives, it finds no available channels. Note that one channel is not being used at this point, but since it was rented in advance, this RU cannot use it. However, when at the next time a SU arrives, it can use this available channel.

Now, the ideal approach is described in the second part of Fig. 4. In this case, the anticipated rent is triggered just before the arrival of the RU in order to cope with the arrival of a PU that causes a SU to perform a spectrum handoff. Thus, under the ideal anticipated approach, a new SU does not trigger the anticipated channel rent. As such, the time that the SS rents a channel is reduced compared to the practical anticipated approach, i.e., resource wastage of rented channel is minimized. Nonetheless, there is still some time during which the rented channels are not used in order to anticipate the arrival of a $\mathrm{SU}$ in spectrum handoff. In this manner, secondary forced termination probability is reduced.

\subsection{Teletrafic analysis}

In this section, a connection-level analytical model for the performance evaluation of CCRN with delay-sensitive traffic is developed. A three-dimensional birth and death process is used to obtain the probability distribution of primary and secondary users in the system. Let us define $\mathbf{e}=\{m, n\}$ and $\mathbf{b}=\left\{b_{m}, b_{n}\right\}$ as arrays that represent the number of PUs in the PS and the number of SUs in the SS, and the number of channels required by each type of user, respectively. For analytical purposes and generalization, it is considered that each type of user can have different bandwidth requirements.

For each leasing strategy, the set of feasible states, $\Omega$, can be built as follows:

\subsubsection{Permanent leasing strategy}

For the PLS, the set of feasible states is given by the following formula:

$\Omega=\left\{(l, m, n) \mid 0 \leq \mathbf{b} \cdot \mathbf{e} \leq N+R \cap m b_{m} \leq N \cap n b_{n} \leq N+R-\lfloor r\rfloor \cap S_{S U} \leq R\right\}$

The first condition in the right side of (1) stablishes that the number of resources in use by PUs and SUs cannot be higher than the total number of resources assigned to the PS and SS sub-networks. The second condition deals with stablishing that the resources used by PUs cannot be higher than the total number of resources assigned to the PS sub-network (since PUs cannot use the leased resources). The third condition guarantees that, considering the use of channel reservation for interrupted secondary calls, the resources used by the SUs can be taken from the ones assigned to the PS and the RS sub-networks. The last condition considers that the number of leased channels by SUs cannot be higher than $R$. Also, note that the number of leased channels is fixed and equal to $R$.

\subsubsection{Dynamical and anticipated leasing}

For the dynamic and anticipated spectrum leasing strategies, the set of valid states is given by the following expression:

$$
\Omega=\begin{aligned}
& \left\{(l, m, n) \mid 0 \leq \mathbf{b} \cdot \mathbf{e} \leq N+R \cap\left(S_{S U}+l b_{l}\right)\right. \\
& \left.\leq K \cap m b_{m} \leq N \cap n b_{n} \leq N+R-\lfloor r\rfloor \cap S_{S U} \leq R\right\}
\end{aligned}
$$

where $S_{S U}=\max (\mathbf{b} \cdot \mathbf{e}-N, 0)$. In $(2),\left(S_{S U}+l b_{l}\right)$ denotes the total number of channels in the RS sub-network used by RUs and SUs.

The first condition in the right side of (2) restrains the maximum number of simultaneously used channels for PUs and SUs in the PS and SS sub-networks. The second condition restrains the maximum number of channels used by SUs and RUs in the RS sub-network. The third (fourth) condition bounds the maximum number of simultaneous channels used by ongoing primary (secondary) sessions. The fifth condition limits the maximum number of leasing channels for (new or interrupted) secondary calls.

Contrary to PLS, in this strategy, the number of leased channels is variable. This is reflected in the second 

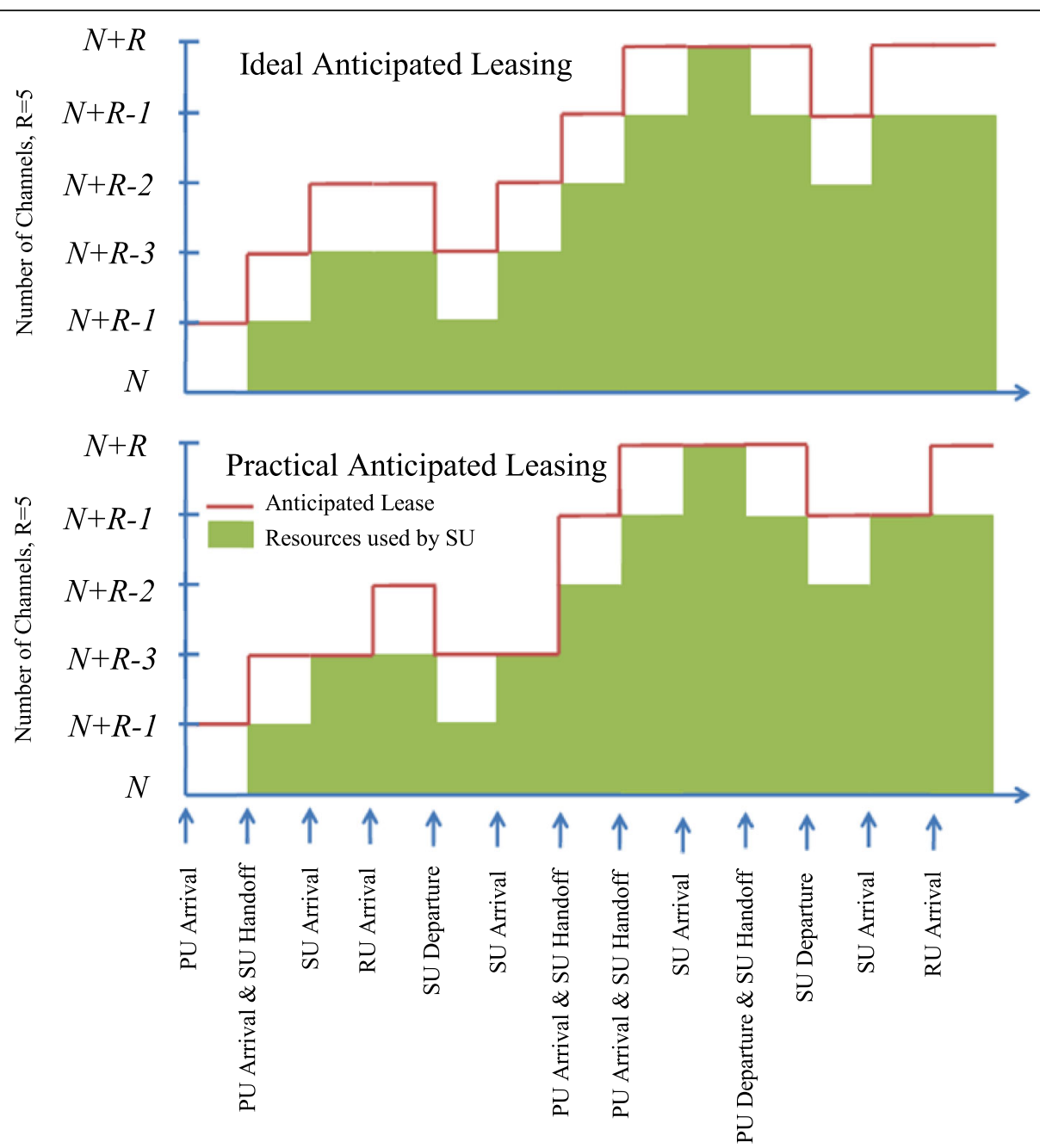

Fig. 4 Timeline of events in the anticipated leasing strategies

condition in the right side of (2). The remaining conditions have the same meaning as in the permanent leasing strategy.

Now, the transition rates between valid system states are developed. Note that, for the PLS, the transition rates described in this section are the same as for the DLS with the following differences: for the former, $R_{\max }$ $=R$, because there are always $R$ channels leased permanently, while for the latter, $R_{\max }=\min \left(R, K-l b_{l}\right)$, because the number of leased channels changes according to the channel utilization of the PS and SS.

When the system is in state $(l, m, n)$, it moves to the following states:

- $(l+1, m, n)$ when a user arrives to the RU subnetwork. This happens with arrival rate $a_{l}(l, m, n)$. For the dynamic leasing scheme, the birth rate is given by (3), while for the anticipated leasing scheme is given by (4). It can be seen that, for the dynamic leasing scheme, a RU can be accepted only if the total number of channels used by SUs and RUs in the RS sub-network is less than $K-b_{l}$. c additional condition has to be met: the arrival of a RU is accepted if the total number of ongoing RUs

plus ongoing SUs in the leasing network has not exceeded $K$ resources.

$a_{l}(l, m, n)=\left\{\begin{array}{lll}\lambda_{l} & ; & l \geq 0,\left(S_{\mathrm{SU}}+l b_{l}\right) \leq K-b_{l} \\ 0 \quad ; & \text { otherwise }\end{array}\right.$ $a_{l}(l, m, n)=\left\{\begin{array}{ccl}\lambda_{l} & ; \quad l \geq 0 \cap\left(\left(\min \left(S_{\mathrm{SU}}+b_{n}, R\right)+l b_{l}\right) \leq K-b_{l} \cap \mathbf{b} \cdot \mathbf{e} \geq N\right) \\ 0 & ; & \text { otherwise }\end{array}\right.$ 
- $(l, m+1, n)$ when a PU arrives to the PS subnetwork and none of the ongoing secondary calls is forced to terminate. This happens with rate:

$a_{m 1}(l, m, n)= \begin{cases}\lambda_{m} & ; m \geq 0 \cap \mathbf{b} \cdot \mathbf{e} \leq N+R_{\max }-b_{m} \cap m b_{m} \leq N-b_{m} \\ 0 & ; \text { otherwise }\end{cases}$

In this case, the new primary arrival is accepted if the number of occupied resources assigned to PUs is lower or equal than $\left(N-b_{m}\right)$ and none of the ongoing secondary sessions is forced to terminate, that is, there exist enough resources in either the PS or the RS sub-networks to handle all ongoing SU's calls after the new primary arrival is accepted in the system.

- $(l, m+1, n-i)$ when a PU arrives to the PS subnetwork and at least one ongoing secondary session is forced to terminate. This happens with rate:

$$
\begin{gathered}
a_{m 2}(l, m, n)=\left\{\begin{array}{lll}
\lambda_{m} & ; & m \geq 0 \cap \mathbf{b} \cdot \mathbf{e}>N+R_{\max }-b_{m} \cap m b_{m} \leq N-b_{m} \\
0 & ; & \text { otherwise }
\end{array}\right. \\
i=1, \ldots,\left\lceil\left\lceil\frac{b_{m}}{b_{n}}\right\rceil\right.
\end{gathered}
$$

In this case, there are not enough resources in the PS and RS sub-networks to handle all the ongoing secondary calls at the moment the new primary request arrives to the system (that is, $\mathbf{b} \cdot \mathbf{e}>N$ $\left.+R_{\max }-b_{m}\right)$.

- $(l, m, n+1)$ when a SU arrives to the CCRN. This happens with rate:

$a_{n}(l, m, n)= \begin{cases}\lambda_{n} & ; n \geq 0 \cap \mathbf{b} \cdot \mathbf{e} \leq N+R_{\max }-\lfloor r\rfloor-1-b_{n} \cap S_{\mathrm{SU}} \leq R-b_{n} \\ \left(1-P_{r}\right) \lambda_{n} & ; n \geq 0 \cap \mathbf{b} \cdot \mathbf{e}=N+R_{\max }-\lfloor r\rfloor-b_{n} \cap S_{\mathrm{SU}} \leq R-b_{n} \\ 0 & ; \text { otherwise }\end{cases}$

A SU session is accepted in the system when there exists enough idle resources in either the PS or RS as noted by the second and third conditions of the first and second rows of the right side of (8). As mentioned before, because of the fractional reservation mechanism, the incoming $\mathrm{SU}$ call is blocked with probability $P_{r}=r-\lfloor r\rfloor$ and it is accepted with probability $\left(1-P_{r}\right)$. It is important to note that the resources used by the SUs on the RS sub-network are bounded by $R$, which denotes the maximum number of leasing channels that can be occupied by SUs on the leasing network.

- $(l-1, m, n)$ when a RU finishes its call. This occurs with rate:

$$
d_{l}(l, m, n)=\left\{\begin{array}{lll}
l \mu_{l} & ; & l>0 \cap\left(S_{\mathrm{SU}}+l b_{l}\right) \leq K \\
0 & ; & \text { otherwise }
\end{array}\right.
$$

This event happens when there are $l$ ongoing RUs occupying $l b_{l}$ of the $K$ channels in the RS sub-network. Thus, this event will happen with rate $l \mu_{l}$ which depends on the actual number of RUs in the system: a higher number of RUs implies a higher death rate.

- $(l, m-1, n)$ when a PU leaves the PS. This happens with rate:

$$
d_{m}(l, m, n)= \begin{cases}m \mu_{m} & ; \quad m>0 \cap b_{m} m \leq N \\ 0 \quad ; \quad \text { otherwise }\end{cases}
$$

This event happens when there are $m$ ongoing PUs occupying $\left(m b_{m}\right)$ of the $N$ channels in the PS sub-network. Thus, this event will happen with rate $m \mu_{m}$ which depends on the actual number of PUs in the system: a higher number of PUs implies a higher death rate.

- $(l, m, n-1)$ when a SU finishes its call. This happens with rate:

$$
d_{n}(l, m, n)=\left\{\begin{array}{cc}
n \mu_{n} \quad ; \quad n>0 \cap n b_{n} \leq N+R-r \cap \mathbf{b} \cdot \mathbf{e} \leq N+R_{\max } \\
\quad-\lfloor r\rfloor \cap S_{\mathrm{SU}} \leq R \cap\left(S_{\mathrm{SU}}+l b_{l}\right) \leq K 0 \\
; \quad \text { otherwise }
\end{array}\right.
$$

This event happens when there are $n$ SUs, each one of them occupying $b_{n}$ channels in either the PS or RS. Thus, this event will happen with rate $n \mu_{n}$; as such, a higher number of ongoing secondary calls implies a higher death rate.

From this description, the balanced equation can be written as: 


$$
\begin{aligned}
& {\left[a_{l}(l, m, n)+a_{m 1}(l, m, n)+a_{m 2}(l, m, n)+a_{n}(l, m, n)+d_{l}(l, m, n)+d_{m}(l, m, n)+d_{n}(l, m, n)\right] P(l, m, n)} \\
& =a_{l}(l-1, m, n) P(l-1, m, n)+a_{m 1}(l, m-1, n) P(l, m-1, n)+a_{n}(l, m, n-1) P(l, m, n-1) \\
& +d_{l}(l+1, m, n) P(l+1, m, n)+d_{m}(l, m+1, n) P(l, m+1, n)+d_{n}(l, m, n+1) P(l, m, n+1) \\
& +\sum_{i=1}^{\left[\frac{b_{m}}{b_{n}}\right]}\left[a_{m 2}(l, m-1, n+i) P(l, m-1, n+i)\right]
\end{aligned}
$$

where rates $a_{l}(l, m, n)$ and $d_{l}(l+1, m, n)$ are true for the DLS and PLS and $P(l, m, n)$ is the probability in steady state for being in the state $(l, m, n)$. Indeed, for the PLS, there is no impact on the arrival or departure of users in the RS sub-network. Building on this, the blocking probability under the PLS can be computed as:

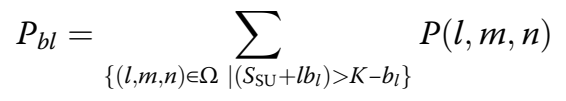

On the other hand, the blocking probability of RUs, PUs, and SUs under the DLS can be computed by (14), (15), and (16), respectively.

$$
P_{b l}=\sum_{\{(l, m, n) \in \Omega} \sum_{\left.\mid\left(\left(S_{\mathrm{SU}}+l b_{l}\right)>K-b_{l} \cap \mathbf{b} \cdot \mathbf{e}<N\right) \cup\left(\left(\min \left(S_{\mathrm{SU}}+b_{n}, R\right)+l b_{l}\right)>K-b_{l} \cap \mathbf{b} \cdot \mathbf{e} \geq N\right)\right\}} P(l, m, n)
$$

$$
\begin{gathered}
P_{b m}=\sum_{\left\{(l, m, n) \in \Omega \mid m b_{m}>N-b_{m}\right\}} P(l, m, n) \\
P_{b n}=\sum_{\left\{(l, m, n) \in \Omega \mid \mathbf{b} \cdot \mathbf{e}>N+R-r-1-b_{n} \cap \mathbf{b} \cdot \mathbf{e}<N+R_{\max }-\lfloor r\rfloor-b_{n} \cap S_{\mathrm{SU}} \leq R\right\}} P(l, m) \\
+\sum_{\left\{(l, m, n) \in \Omega \mid \mathbf{b} \cdot \mathbf{e}=N+R_{\max }-\lfloor r\rfloor-b_{n} \cap S_{\mathrm{SU}} \leq R\right\}} P(l, m, n)
\end{gathered}
$$

New call blocking occurs when there are no enough resources to carry the service of a certain type of user in the system. Generally, for PUs, new call blocking occurs when all $N$ resources are being used by PUs. New call blocking for SUs occurs when the $N+R$ resources are being used, while new call blocking for RUs occurs when the $K$ resources of the RS sub-network are being occupied either by SUs or RUs.

Forced termination occurs when a $\mathrm{SU}$ is interrupted by a PU and cannot be allocated in another channel of either the PS or the RS, that is, when a new primary call arrives to the system, all the resources from the PS and the RS are being used (as denoted in $m \geq 0 \cap \mathbf{b} \cdot \mathbf{e}>N+$ $\left.R_{\max }-b_{m} \cap m b_{m} \leq N-b_{m}\right)$, and at least there exist one $\mathrm{SU}$ in the system, a certain number of SUs will be forced to terminate: $\left(\left\lceil\frac{\mathbf{b} \cdot \mathbf{e}-\left(N+R_{\max }-b_{m}\right)}{b_{n}}\right\rceil\right)$. Thus, the probability that a secondary call is forced to terminate can be computed by (17).

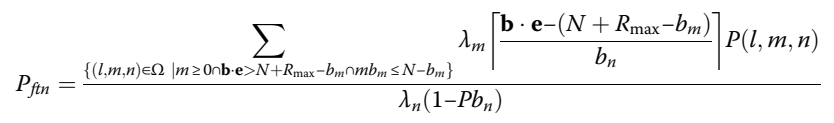

As stated before, the PLS is the simplest leasing strategy but has the drawback of the underutilization of the leased resources. Conversely, the DLS and ALS are more complex, but there is a better utilization of the leased resources. In the context of CCRNs, it is important to have a performance metric related to the cost of leasing a channel. This metric is called the cost per Erlang of capacity. To calculate the cost per Erlang of capacity, the average number of channels actually being used $(E\{L\})$ needs to be calculated. This is the aim of the following subsections.

a) Dynamic leasing strategy:

For the DLS, the average number of channels being used can be computed by averaging the number of resources rented by the SUs in state $(l, m, n)\left(S_{R}\right)$ and pondering by the probability of being in that state. Mathematically, this parameter can be described as follows:

$$
E\{L\}=\sum_{\{(l, m, n) \in \Omega} S_{\left.\mid \mathbf{b} \cdot \mathbf{e} \geq N \cap S_{\mathrm{su}} \leq R\right\}} P(l, m, n)
$$

On the other hand, the arrival rate of SUs to the RS is denoted by $\lambda_{R}$ and can be obtained by averaging the arrival rates at the state $(l, m, n)$ pondered by their respective state probabilities and taking into account the number of channels that where leased and reserved $\left(I_{1}, I_{2}\right)$. Mathematically, this arrival rate can be expressed as follows:

$$
\lambda_{R}=\sum_{\{(l, m, n) \in \Omega\}}\left(a_{m 1}(l, m, n) I_{1}+a_{n}(l, m, n) I_{2}\right) P(l, m, n)
$$

where 
$I_{1}=\left\{\begin{array}{lll} & 1 & ; \quad \mathbf{b} \cdot \mathbf{e} \geq N \cap \min \left(K-l b_{l}, R\right) \leq R-b_{n} \cap S_{\mathrm{SU}} \leq R-b_{n} \cap\left(n-\mathrm{SU}_{r}\right)>0 \\ 0 & ; & \text { otherwise }\end{array}\right.$

$I_{2}=\left\{\begin{array}{ccl}1 & ; \quad \mathbf{b} \cdot \mathbf{e} \geq N \cap \min \left(K-l b_{l}, R\right) \leq R-b_{n} \cap S_{\mathrm{SU}} \leq R-b_{n} \\ 0 \quad ; & \text { otherwise }\end{array}\right.$

$I_{1}$ and $I_{2}$ are indicating functions. $I_{1}\left(I_{2}\right)$ indicates when a channel is being leased every time a primary (secondary) arrival occurs.

From (18) and (19), it is easy to calculate the average time that SUs occupy resources at the RS. This can be done by applying Little's theorem, which states that:

$$
E\{W \mid W>0\}=\frac{E\{L\}}{\lambda_{R}}
$$

\section{b) Anticipated leasing strategy:}

For the practical approach, the average number of channels being used can be computed as follows:

$$
E\{L\}=\sum_{\{(l, m, n) \in \Omega} S_{\left.\mid \mathbf{b} \cdot \mathbf{e} \geq N \cap S_{\mathrm{SU}} \leq R\right\}} S_{R} P(l, m, n)
$$

where $S_{R}=\min \left(S_{\mathrm{SU}}+b_{n}, R\right)$ is the number of resources rented by SUs.

On the other hand, the arrival rate of SUs to the RU sub-network considering the practical anticipated approach can be computed by

$$
\lambda_{R}=\sum_{\{(l, m, n) \in \Omega\}}\left[a_{m 1}(l, m, n) I_{3}+a_{n}(l, m, n) I_{4}\right] P(l, m, n)
$$

where

$$
I_{3}=\left\{\begin{array}{rll}
2 & ; \quad & \mathbf{b} \cdot \mathbf{e} \geq N \cap \min \left(K-l b_{l}, R\right) \leq R-2 b_{n} \cap S_{\mathrm{SU}} \leq R-2 b_{n} \cap\left(n-\mathrm{SU}_{r}\right)>0 \\
1 & ; \quad \mathbf{b} \cdot \mathbf{e} \geq N \cap \min \left(K-l b_{l}, R\right) \leq R-b_{n} \cap S_{\mathrm{SU}} \leq R-b_{n} \cap\left(n-\mathrm{SU}_{r}\right)>0 \\
0 & ; \quad \text { otherwise }
\end{array}\right.
$$

$$
I_{4}=\left\{\begin{array}{rll}
2 & ; \quad \mathbf{b} \cdot \mathbf{e} \geq N \cap \min \left(K-l b_{l}, R\right) \leq R-2 b_{n} \cap S_{\mathrm{SU}} \leq R-2 b_{n} \\
1 & ; \quad \mathbf{b} \cdot \mathbf{e} \geq N \cap \min \left(K-l b_{l}, R\right) \leq R-b_{n} \cap S_{\mathrm{SU}} \leq R-b_{n} \\
0 \quad ; \quad \text { otherwise } &
\end{array}\right.
$$

In (25) and (26), it can be seen that whenever a PU or a SU is accepted in the PS and resources are still available, an additional channel is requested. From this, it is clear that the additional channel is not used immediately and is assigned to a SU only when needed. Hence, the arrival of a PU or SU triggers this mechanism because both types of events consume idle resources in the PS.
For the ideal approach, the average number of channels being used can be computed as follows:

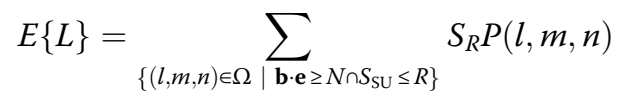

On the other hand, the arrival rate of SUs to the RU sub-network considering the ideal anticipated approach can be computed as:

$$
\lambda_{R}=\sum_{\{(l, m, n) \in \Omega\}}\left[\left\{a_{m 1}(l, m, n) I_{5}+\left(a_{n}(l, m, n)+a_{l}(l, m, n)\right) I_{6}\right\}\right] P(l, m, n)
$$

where

$I_{5}=\left\{\begin{array}{rll}2 & ; \quad \mathbf{b} \cdot \mathbf{e} \geq N \cap \min \left(K-l b_{l}, R\right) \leq R-2 b_{n} \cap S_{\mathrm{SU}} \leq R-2 b_{n} \cap\left(n-\mathrm{SU}_{r}\right)>0 \\ 1 & ; \quad \mathbf{b} \cdot \mathbf{e} \geq N \cap \min \left(K-l b_{l}, R\right) \leq R-b_{n} \cap S_{\mathrm{SU}} \leq R-b_{n} \cap\left(n-\mathrm{SU}_{r}\right)>0 \\ 0 & ; \quad \text { otherwise }\end{array}\right.$

$I_{6}=\left\{\begin{array}{cl}1 \quad ; \quad \mathbf{b} \cdot \mathbf{e} \geq N \cap \min \left(K-l b_{l}, R\right) \leq R-b_{n} \cap S_{\mathrm{SU}} \leq R-b_{n} \\ 0 \quad ; \quad \text { otherwise }\end{array}\right.$

When comparing the practical and ideal approach, it should be noted that in the ideal approach, not only a channel does have to be rented upon the arrival of a SU or PU in the PS, but also a channel has to be rented just before the arrival of a RU in the system. Clearly, the implementation of the ideal scenario is not feasible because the arrival of RUs cannot be predicted. However, the ideal scenario is investigated here as an upper maximum theoretical limit to study the best performance of the ALS. Finally, it is important to note that, for the case when $r$ and $R$ take real values, these transition probabilities are different from the ones previously presented. In Appendix, the modified rates are described for the PLS.

On the other hand, it is interesting to analyze and evaluate the case of multiple leasing sub-networks and/or multiple sources of spectrum owners. In the developed analysis of the present paper, a state variable is used to represent the number of users in each considered sub-network (i.e., the number of RUs, PUs, and SUs is represented by state variables $l, m$, and $n$, respectively). For the case of multiple leasing sub-networks and/or multiple sources of spectrum owners, the teletraffic analysis can be extended by using one additional state variable to identify the number of users in each additional sub-network: either leasing sub-network or any other considered source of spectrum owner. To determine state transition rates, it is necessary to know the system state, arrival rates to each sub-network, and users' departure rates. 
With the aforementioned analysis, it is possible to establish a series of theorems that can describe the behavior of the system in terms of the Erlang capacity. It is important to take into account this behavior to obtain the optimal values of certain parameters that satisfy the QoS of the system.

Theorem 1 The Erlang capacity for DLS and ALS monotonically decreases with the offered traffic of the RUs.

Proof Let us consider that, for a given value of the traffic load of the RUs $\left(a_{\mathrm{RU}}\right)$, a CRN is operating at the optimal configuration, that is, the CRN uses the optimal number of reserved channels $r^{\text {(opt) }}$ such as the offered traffic load of the SUs $\left(a_{\mathrm{SU}}{ }^{(\mathrm{opt})}\right)$ is equal to the Erlang capacity of the SS. Under this configuration, the call blocking probability $P_{\mathrm{bn}}$ and the forced termination $P_{\mathrm{ftn}}$ probabilities of SUs achieve their maximum acceptable values, that is:

$$
\begin{aligned}
& P_{\mathrm{bn}}\left(a_{\mathrm{SU}}{ }^{(\mathrm{opt})}, a_{\mathrm{RU}}, r^{(\mathrm{opt})}\right)=2 \% \\
& P_{\mathrm{ftt}}\left(a_{\mathrm{SU}}{ }^{(\mathrm{opt})}, a_{\mathrm{RU}}, r^{(\mathrm{opt})}\right)=2 \%
\end{aligned}
$$

The dependency of $P_{\mathrm{bn}}$ and $P_{\mathrm{ftn}}$ on the offered traffic load of SUs, the offered traffic load of RUs, and the number of reserved channels has been explicitly indicated in (31).

Let us consider that the offered traffic load of RUs increases from $a_{\mathrm{RU}}$ to $a_{\mathrm{RU}}+\Delta a_{\mathrm{RU}}$. Then, the resource occupation in the RS increases; therefore, the probability of finding an available leasing resource decreases.

This phenomenon causes that $P_{\mathrm{bn}}$ and $P_{\mathrm{ftn}}$ increase above their respective maximum acceptable values, that is:

$$
\begin{aligned}
& P_{\mathrm{bn}}\left(a_{\mathrm{SU}}{ }^{(\mathrm{opt})}, a_{\mathrm{RU}}+\Delta a_{\mathrm{RU}}, r^{(\mathrm{opt})}\right)>2 \% \\
& P_{\mathrm{ftn}}\left(a_{\mathrm{SU}}{ }^{(\mathrm{opt})}, a_{\mathrm{RU}}+\Delta a_{\mathrm{RU}}, r^{(\mathrm{opt})}\right)>2 \%
\end{aligned}
$$

In order to decrease the values of $P_{\mathrm{bn}}$ and $P_{\mathrm{ftn}}$ to achieve their maximum acceptable values, it is necessary to decrease the offered traffic load of SUs to a new optimal value $a_{\mathrm{SU}}{ }^{\text {(new) }}$ and recalculate the new optimal value of reserved channels $r^{\text {(new) }}$. Then,

$$
\begin{aligned}
& P_{\mathrm{bn}}\left(a_{\mathrm{SU}}{ }^{(\text {new })}, a_{\mathrm{RU}}+\Delta a_{\mathrm{RU}}, r^{(\text {new })}\right)=2 \% \\
& P_{\mathrm{ftn}}\left(a_{\mathrm{SU}}{ }^{(\text {new })}, a_{\mathrm{RU}}+\Delta a_{\mathrm{RU}}, r^{(\text {new })}\right)=2 \%
\end{aligned}
$$

where $a_{\mathrm{SU}}{ }^{\text {(new) }}<a_{\mathrm{SU}}{ }^{\text {(opt) }}$. Thus, it has been proven that the Erlang capacity of SUs decreases when the offered traffic load of RUs increases.

Theorem 2 As the value of the maximum number of channels allowed being leased $\left(R_{\max }\right)$ increases, the achieved Erlang capacity increases as well.
Proof Let us consider the optimal configuration for the system, that is, when the SUs offered traffic load is equal to the Erlang capacity of the SS. Additionally, consider a maximum number of channels allowed to be leased, $R_{\max }$. Then, the call blocking $P_{\mathrm{bn}}$ and the forced termination $P_{\mathrm{ftn}}$ probabilities of SUs achieve their maximum acceptable values, that is:

$$
\begin{aligned}
& P_{\mathrm{bn}}\left(a_{\mathrm{SU}}{ }^{(\mathrm{opt})}, a_{\mathrm{RU}}, R_{\mathrm{max}}, r^{(\mathrm{opt})}\right)=2 \% \\
& P_{\mathrm{ftn}}\left(a_{\mathrm{SU}}{ }^{(\mathrm{opt})}, a_{\mathrm{RU}}, R_{\mathrm{max}}, r^{(\mathrm{opt})}\right)=2 \%
\end{aligned}
$$

Notice that (34) explicitly indicates the dependency of $P_{\mathrm{bn}}$ and $P_{\mathrm{ftn}}$ on the offered traffic load of the SUs, the offered traffic load of the RUs, and the number of reserved channels.

Let us consider that the maximum number of channels allowed being leased increases from $R_{\max }$ to $R_{\max }$ $+\Delta R_{\max }$, then, the probability of finding available resources for secondary calls increases. Therefore, the blocking and forced termination probabilities of $\mathrm{SU}$ tend to decrease below their respective maximum acceptable values, that is:

$$
\begin{aligned}
& P_{\mathrm{bn}}\left(a_{\mathrm{SU}}{ }^{(\mathrm{opt})}, a_{\mathrm{RU}}, R_{\max }+\Delta R_{\max }, r^{(\mathrm{opt})}\right)<2 \% \\
& P_{\mathrm{ftn}}\left(a_{\mathrm{SU}}{ }^{(\mathrm{opt})}, a_{\mathrm{RU}}, R_{\max }+\Delta R_{\max }, r^{(\mathrm{opt})}\right)<2 \%
\end{aligned}
$$

This means that the SS supports a higher optimal traffic load, $a_{\mathrm{SU}}{ }^{\text {(new) }}$, and the optimal value of reserved channels $r^{\text {(new) }}$ must be recalculated. Thus:

$$
\begin{aligned}
& P_{\mathrm{bn}}\left(a_{\mathrm{SU}}{ }^{(\text {new })}, a_{\mathrm{RU}}, R_{\max }+\Delta R_{\max }, r^{(\text {new })}\right)=2 \% \\
& P_{\mathrm{ftn}}\left(a_{\mathrm{SU}}{ }^{(\text {new })}, a_{\mathrm{RU}}, R_{\max }+\Delta R_{\max }, r^{(\text {new })}\right)=2 \%
\end{aligned}
$$

where $a_{\mathrm{SU}}{ }^{\text {(new) }}>a_{\mathrm{SU}}{ }^{\text {(opt) }}$. Hence, it has been proven that the Erlang capacity of the SUs increases when the maximum number of leasing channels increases.

\section{Results and discussion}

The objective of this work is the study and comparison of the PSL, DSL, and ASL strategies; for this reason, a numerical evaluation is presented under different traffic conditions. With this comparison, we are able to benchmark the mathematical models developed in Section 2 in terms of their applicability, preciseness, and robustness. Unless otherwise stated, the system parameters that are used in the plots of this section are the following: $M=15 ; K=15 ; \lambda_{l}=\lambda_{m}$ $=\lambda_{n}=1 / 80$. Two different values for the utilization factor of the primary channels of the PS (i.e., $\rho_{\mathrm{PU}}$ ) 
are considered: 0.1 and 0.2 (to achieve these values of utilization for the PS a 0.05 blocking probability is considered). Two different values for the mean secondary service time relative to the mean primary service time ratio (i.e., $\mu_{\mathrm{m}} / \mu_{\mathrm{n}}$ ) are considered: 1 and 0.1. Two different scenarios relative to the bandwidth requirement for PUs and SUs are considered: the one in which $b_{m}=b_{n}$ and the one in which $b_{m}=$ $3 b_{n}$ (in both scenarios $b_{n}=1$ ). For the secondary QoS metrics, it is considered that the maximum allowed new call blocking probability is equal to 0.02 and the forced terminated probability is equal to 0.002 . System performance is evaluated in terms of maximum Erlang capacity, cost per rented resource per achieved Erlang of capacity, and optimal number of reserved channels.

Figures $5,6,7$, and 8 present the performance evaluation for the permanent leasing strategy (PLS) and our proposed dynamic (DLS) and anticipated (ALS) leasing strategies for different scenarios and system parameters. Specifically, indents (a), (b), and (c) of Figs. 5, 6, 7 and 8 show, respectively, maximum Erlang capacity, cost per rented resource per achieved Erlang of capacity, and the optimal number of reserved channels as function of both utilization factor of primary channels of the leasing network (i.e., $\left.\rho_{\mathrm{RU}}\right)$ and maximum allowed number of rented channels (i.e., $R$ ). The scenario presented in Fig. 5 (i.e., scenario in which $\rho_{\mathrm{PU}}=0.1, \mu_{m} / \mu_{n}=1$, and $b_{m}=$ $b_{n}$.) is taken as the reference one. On the other hand, Figs. 6, 7, and 8 respectively correspond to the following system scenarios: (i) $\rho_{\mathrm{PU}}=0.2, \mu_{m} / \mu_{n}=1$, and $b_{m}=b_{n}$; (ii) $\rho_{\mathrm{PU}}=0.1, \mu_{m} / \mu_{n}=1$, and $b_{m}=3 b_{n}$; (iii) $\rho_{\mathrm{PU}}=0.1, \mu_{m} / \mu_{n}=0.1$, and $b_{m}=b_{n}$; and (iv) $\rho_{\mathrm{PU}}$ $=0.2, \mu_{m} / \mu_{n}=0.1$, and $b_{m}=3 b_{n}$. It is important to state that, with regard to the maximum Erlang capacity and optimal number of reserved channels, practical and ideal anticipated leasing strategies have the same performance. Thus, in indents (a) and (c) of Figs. 5, 6, 7, and 8, the plots labeled "anticipated leasing" corresponds to both practical and ideal anticipated leasing strategies.

Indents (a) of Figs. 5, 6, 7, and 8 show that the both ALS and DLS underperform PLS in terms of maximum Erlang capacity, while the ALS outperforms the DLS. This phenomenon is more notorious as both $R$ and $\rho_{\mathrm{RU}}$ increase. For instance, considering the reference scenario for the case when $\rho_{\mathrm{RU}}=$ 0.9 and $R=4$, Fig. 5a shows that the ALS and DLS achieve, respectively, $15 \%$ and $25 \%$ less traffic than the PLS, while for the same scenario, the ALS achieves $10 \%$ more traffic than the DLS. Also, indent (a) of Figs. 5, 6, 7 and 8 confirm that Erlang capacity increases as $R$ increases. On the other hand, indents (a) of Figs. 5, 6, 7, and 8 show that the maximum Erlang capacity achieved by the PLS is insensitive to $\rho_{\mathrm{RU}}$, while the maximum Erlang capacity is a monotonically decreasing function of $\rho_{\mathrm{RU}}$ for the ALS and DLS as depicted in Theorem 1. As expected, PLS outperforms the other strategies in the possible achievable capacity because of the availability of the resources, while in the other two, available resources cannot be guaranteed all the time. On the other hand, the price that the PLS has to pay is an elevated cost per rented resource per achieved Erlang capacity. In this sense, indents (b) of Figs. 5, 6, 7 and 8 clearly show that the cost per resource per achieved Erlang capacity (hereafter called simply cost per Erlang) is drastically reduced when the ALS or DLS are employed. This effect is more notorious as $R$ increases. For instance, considering the reference scenario for the case when $\rho_{\mathrm{RU}}=0.9$ and $R=4$, Fig. 5b shows that the cost per Erlang under the ALS and DLS are, respectively, 90\% and 95\% smaller than the corresponding cost under the PLS. Also of importance, indents (b) of Figs. 5, 6, 7, and 8 reveal that the cost per Erlang slightly increases as $R$ increases when (both ideal and practical) ALS and DLS strategies are considered as described in Theorem 2. This behavior is due to the fact that under these strategies, resources are rented from the leasing network just at the moment they are needed (this applies for both ideal and practical anticipated and dynamic leasing strategies) or they are rented in advance only when the number of busy channels in the PS is in the critical zone (this applies for both ideal and practical anticipated leasing strategies.) On the other hand, from indents (b) of Figs. 5, 6, 7, and 8 and referring to the anticipated and dynamic leasing strategies, it is observed that the cost per Erlang slightly decreases as $\rho_{\mathrm{RU}}$ increases. This is an intuitive behavior because as $\rho_{\mathrm{RU}}$ increases, the probability that the secondary network acquires (rents) an available resource (when needed) from the leasing network decreases in benefit of cost per Erlang but in detriment of Erlang capacity. Notice from indents (b) of Figs. 5, 6, 7, and 8 that the cost per Erlang is just slightly greater for the anticipated leasing strategies compared against the DLS. In other words, anticipated leasing strategies outperform DLS in terms of Erlang capacity with a negligible penalty in terms of cost per Erlang. Also, indents (b) of Figs. 5, 6, 7, and 8 reveal that our proposed practical ALS achieves nearly the same cost per Erlang compared against the ideal ALS.

On the other hand, from indents (c) of Figs. 5, 6, 7 , and 8 , it is observed that the optimal number of reserved channels is insensitive to changes in the 

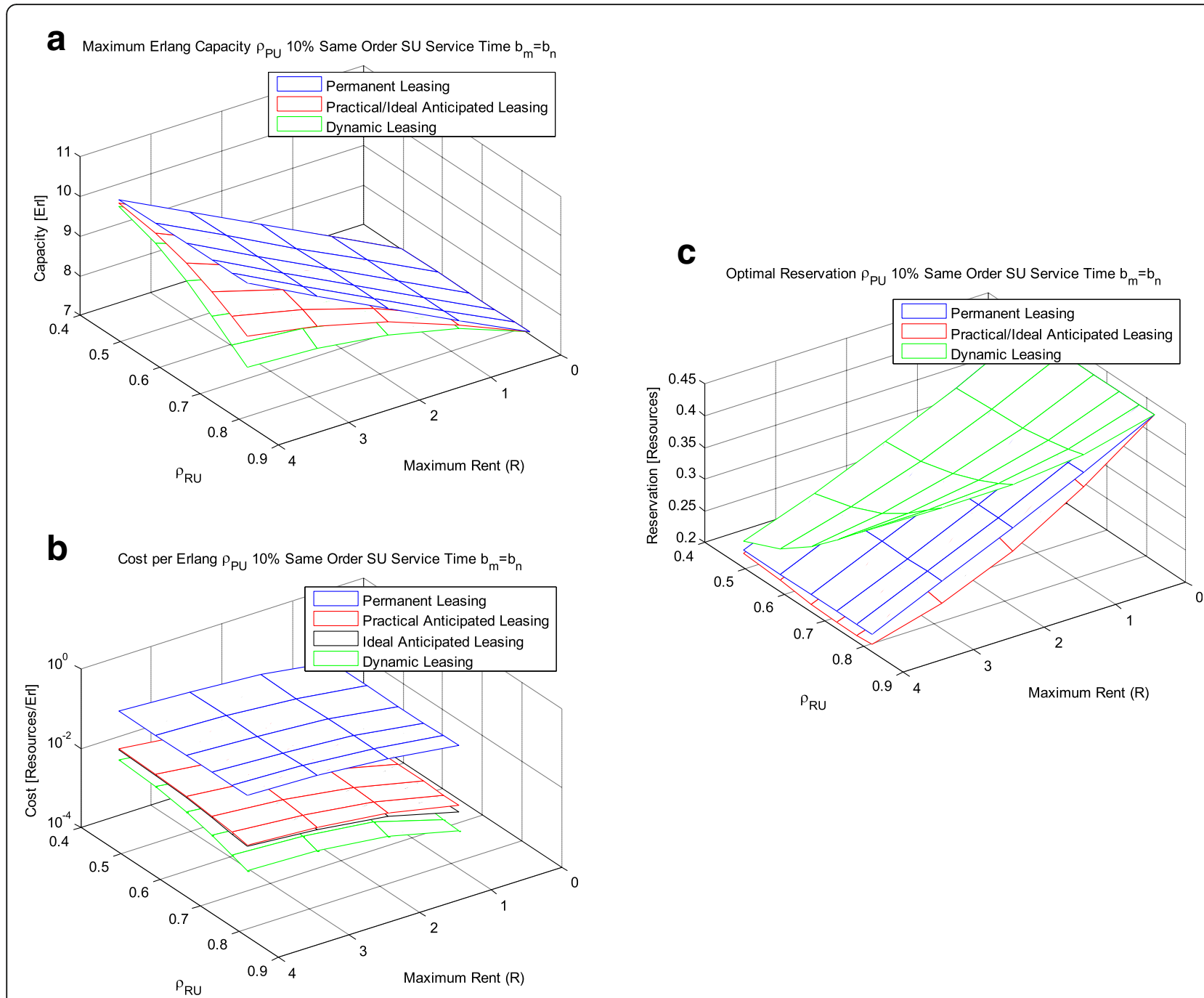

Fig. 5 Performance of spectrum leasing strategies under the system scenario in which $\rho_{\mathrm{PU}}=0.1, \mu_{\mathrm{m}} / \mu_{\mathrm{n}}=1$, and $b_{m}=b_{n}$. a Maximum Erlang capacity, $\mathbf{b}$ cost per rented resource per achieved Erlang capacity, and $\mathbf{c}$ optimal number of reserved channels as function of $R$ and $\rho_{P U}$

$\rho_{\mathrm{RU}}$ when the PLS is considered. Referring to the DLS, indents (c) of Figs. 5, 6, and 7 reveal that, in general terms, as $\rho_{\text {RUincreases, the optimal number }}$ of reserved channels increases. This behavior is more evident as $R$ increases, and it can be explained as follows. Under the DLS, as $\rho_{\mathrm{RU}}$ increases, the probability that a preempted SU (that did not find available resources in the PS) finds an available resource to be rented from the RS decreases in detriment of forced termination probability, consequently, a greater number of reserved channels is needed to compensate this effect. This leads us to the behavior illustrated in indents (c) of Figs. 5, 6, and 7. On the other hand, the behavior of the optimal number of reserved channels as $\rho_{\mathrm{RU}}$ increases under the ALS depends on the particular system conditions. For instance, from Figs. $5 \mathrm{c}$ and $6 \mathrm{c}$, it is observed that, for system conditions in which $\mu_{\mathrm{m}} / \mu_{\mathrm{n}}=1, b_{m}=b_{n}$, and irrespective if $\rho_{\mathrm{RU}}$ is equal to 0.1 or 0.2 , the optimal number of reserved channels slightly decreases as $\rho_{\mathrm{RU}}$ increases under the ALS strategy. This particular behavior can be explained as follows. Under the ALS, as $\rho_{R U}$ increases the probability that the SS rent resources in advance from the leasing network increases in benefit of decreasing the forced termination probability of SUs, consequently, a smaller number of reserved channels for prioritization of ongoing secondary calls over new ones is required. This leads us to the behavior explained above and illustrated in Figs. 5c and 6c. Similar arguments can 


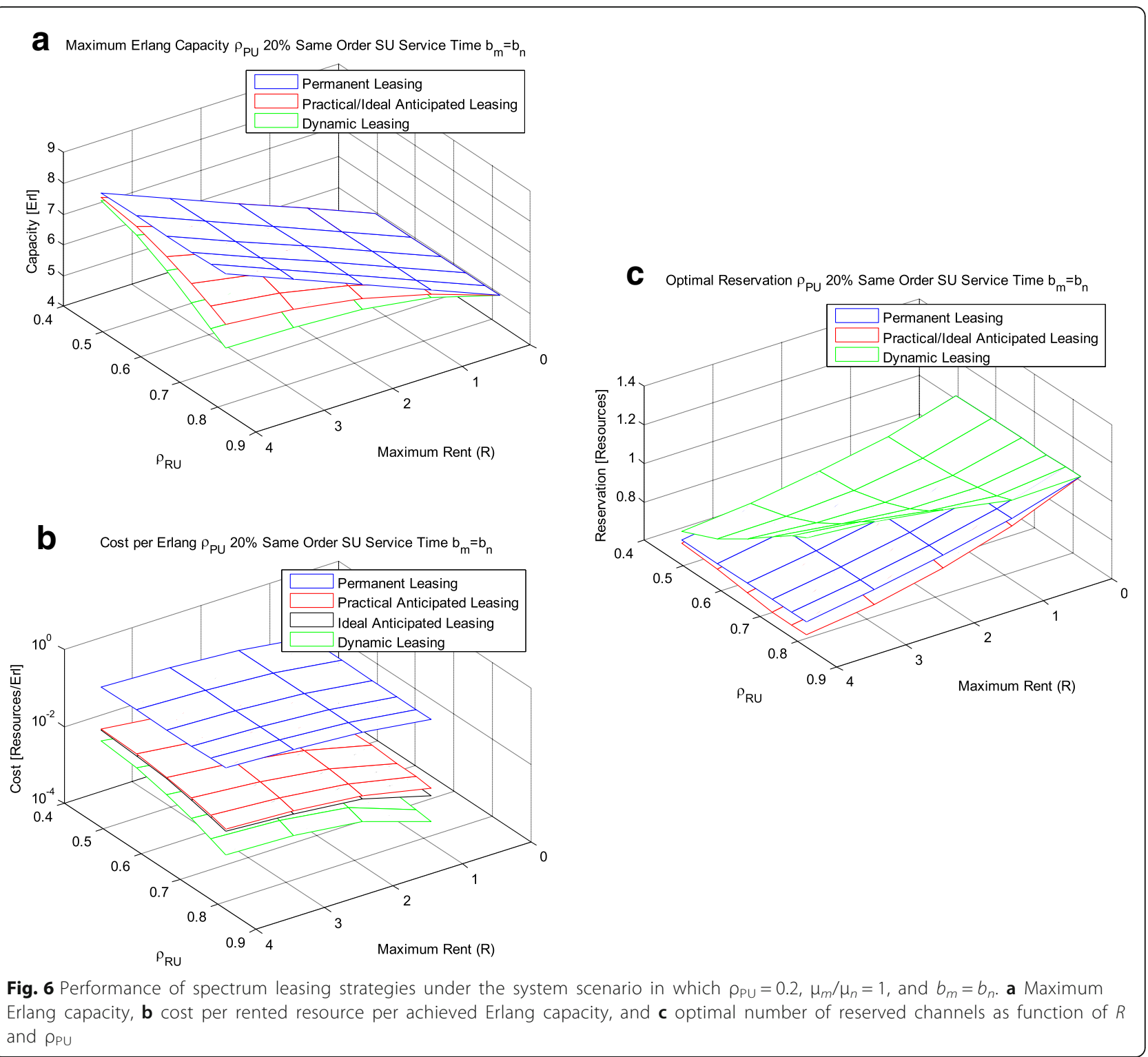

be given to explain the fact that as $R$ increases, the optimal number of reserved channels decreases when the anticipated and permanent leasing strategies are considered under the scenarios represented by Figs. 5c and 6c.

Now, let us investigate the effect of the relative value of the mean secondary service time relative to the mean primary service time (i.e., $\mu_{m} / \mu_{n}$ ). To this end, let us compare Fig. 5 where $\mu_{m} / \mu_{n}=1$ against Fig. 8 where $\mu_{m} / \mu_{n}=0.1$. From the Erlang capacity point of view (Figs. 5a and 8a), it can be seen that as $\mu_{m} / \mu_{n}$ changes from 1 to 0.1 , the maximum Erlang capacity for each strategy also increases. For instance, for the case when $\rho_{\mathrm{RU}}=0.9$ and $R=4$,
Figs. 5a and $8 \mathrm{a}$ show that the Erlang capacity achieved by the PLS, ALS, DLS increases, respectively, $8 \%, 10 \%$, and $16 \%$ as $\mu_{m} / \mu_{n}$ changes from 1 to 0.1 . This behavior obeys to the fact that as $\mu_{m} / \mu_{n}$ goes from 1 to 0.1 , the secondary service times are on average shorter than the primary service times. Thus, in order to achieve the same primary traffic load in both cases, the primary inter-arrival time increases (in the same proportion as $\mu_{m} / \mu_{n}$ decreases) relative to the mean secondary time and, consequently, the forced termination probability decreases in benefit of both blocking and forced termination probabilities. In this manner, system capacity is improved. This fact implies that fewer 

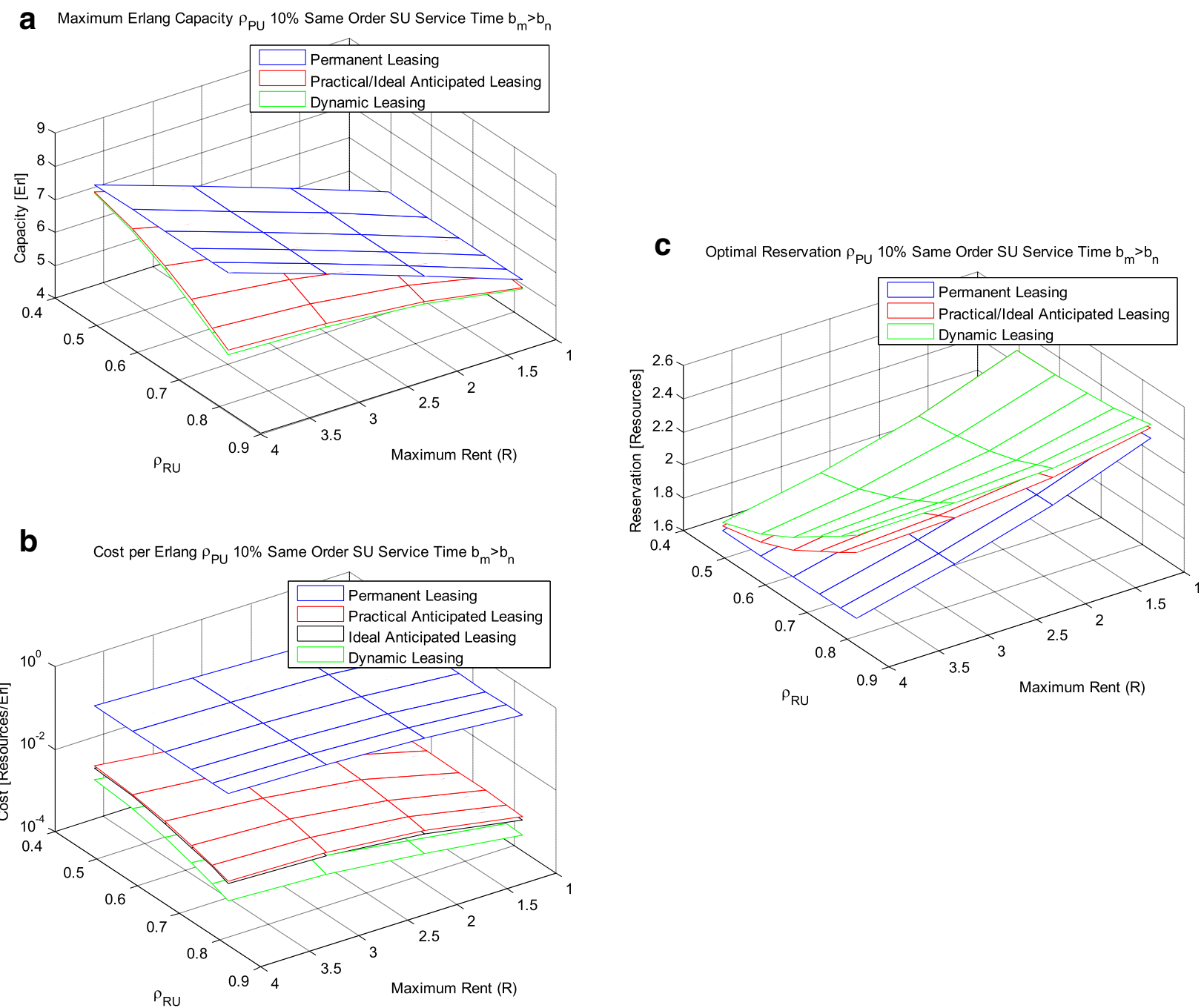

Fig. 7 Performance of spectrum leasing strategies under the system scenario in which $\rho_{\mathrm{PU}}=0.1, \mu_{m} / \mu_{n}=1$, and $b_{m}=3 b_{n}$. a Maximum Erlang capacity, $\mathbf{b}$ cost per rented resource per achieved Erlang capacity, and $\mathbf{c}$ optimal number of reserved channels as function of $R$ and $\rho_{P U}$

reserved channels are required to satisfy the required QoS. For this particular scenario (i.e., $\rho_{\mathrm{PU}}=0.1, \mu_{m} /$ $\mu_{n}=0.1$, and $\left.b_{m}=b_{n}\right)$, Fig. 8a shows that no reserved channels are needed in order to maximize system Erlang capacity.

Now, let us investigate the effect of the utilization factor of the primary network (i.e., $\rho_{\mathrm{PU}}$ ) versus the non-homogeneous bandwidth requirement for PUs and SUs (i.e., $b_{m} / b_{n}$ ). To this end, let us compare Fig. 5 where $\rho_{\mathrm{PU}}=0.1$ and $b_{m}=b_{n}$ against both Fig. 6 where $\rho_{\mathrm{PU}}=0.1$ and Fig. 7 where $b_{m}=3 b_{n}$. From the Erlang capacity point of view, when comparing Fig. $5 \mathrm{a}$ against Figs. $6 \mathrm{a}, 7 \mathrm{a}$, it is observed that as $\rho_{\mathrm{PU}}$ $\left(b_{m} / b_{n}\right)$ changes from 0.1 (1.0) to 0.2 (3.0), the maximum Erlang capacity achieved by the different spectrum leasing strategies decreases. This is an expected behavior because when either $\rho_{\mathrm{PU}}$ or $b_{m} / b_{n}$ increases, the forced termination probability of secondary sessions increases in detriment of the Erlang capacity. This also implies that a greater number of reserved channels are required to satisfy the required QoS for the SUs as it is shown in Figs. 6c and 7c (compared against Fig. 5c).

\section{Conclusions}

In this work, two dynamic spectrum leasing strategies with anticipated resource leasing are proposed to control the effects of the random nature of resource availability in coordinated cognitive radio networks with fixed-rate 
a Maximum Erlang Capacity $\rho_{\mathrm{PU}} 10 \%$ One Order Smaller SU Service Time $\mathrm{b}_{\mathrm{m}}=\mathrm{b}_{\mathrm{n}}$

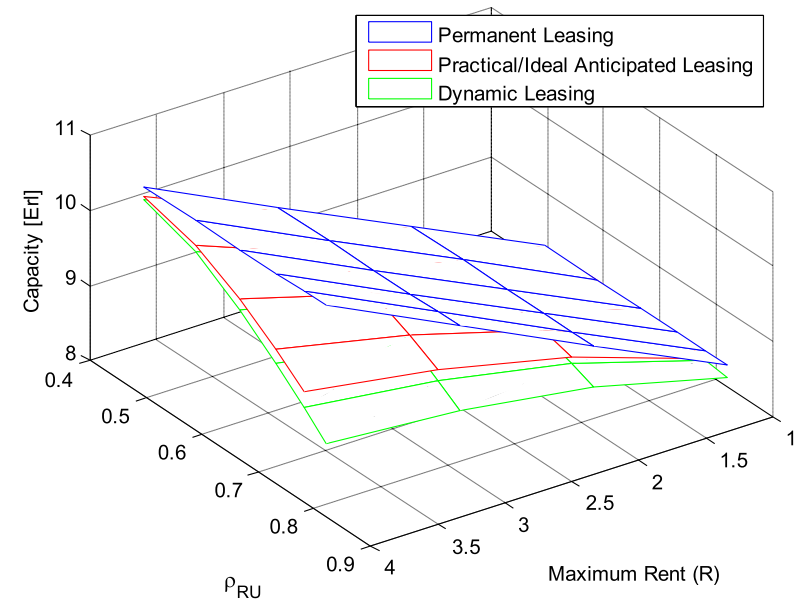

b Cost per Erlang $\rho_{\mathrm{PU}} 10 \%$ One Order Smaller SU Service Time $b_{m}=b_{n}$
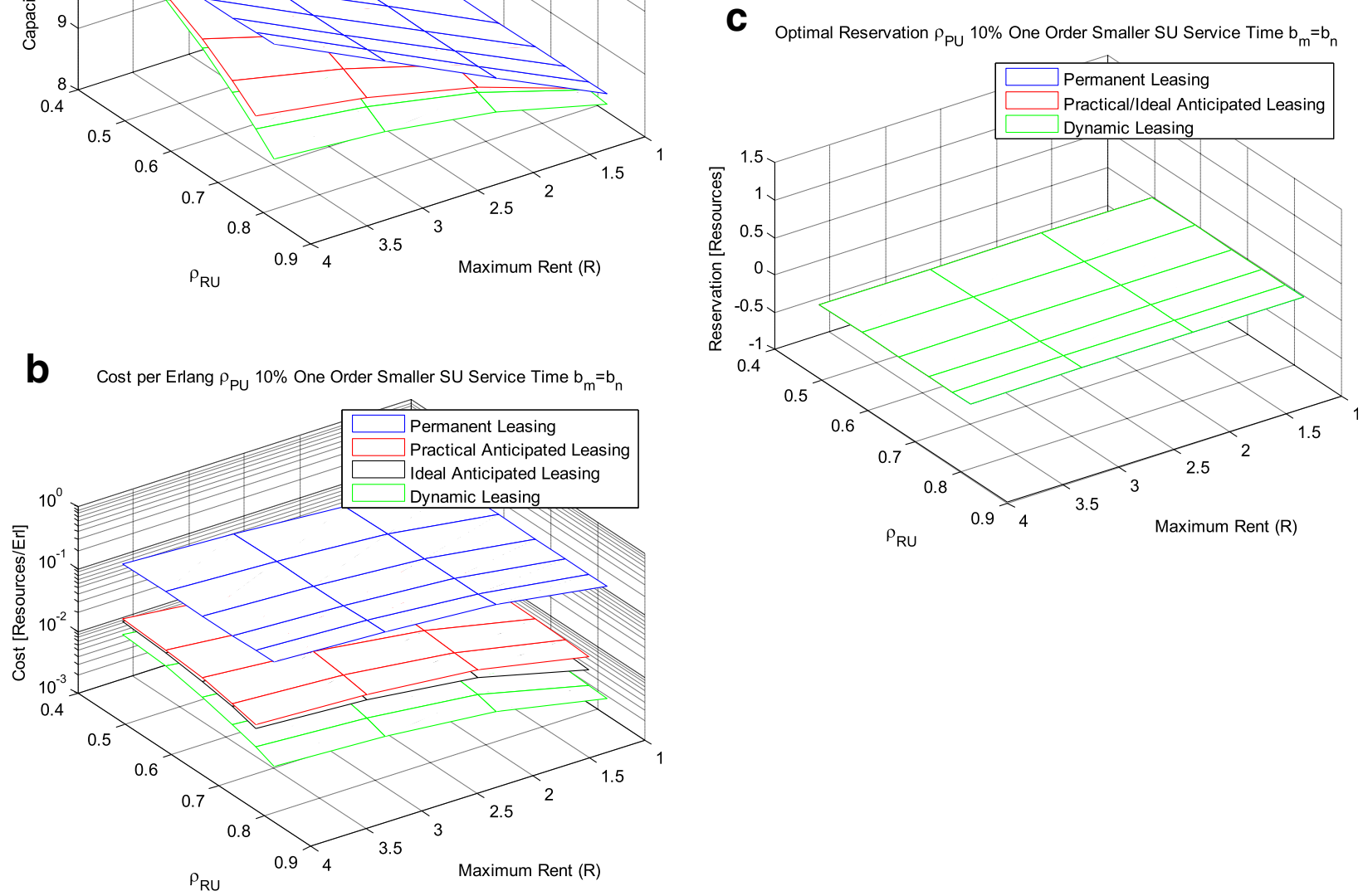

Fig. 8 Performance of spectrum leasing strategies under the system scenario in which $\rho_{\mathrm{PU}}=0.1, \mu_{m} / \mu_{n}=0.1$, and $b_{m}=b_{n}$. a Maximum Erlang capacity, $\mathbf{b}$ cost per rented resource per achieved Erlang capacity, and $\mathbf{c}$ optimal number of reserved channels as function of $R$ and $\rho_{P U}$

traffic and hard-delay requirements. It is also important to remark that, although our numerical results are extracted for particular scenarios, with a certain set of parameter values, our contribution reveals important design aspects to be considered when leasing strategies are employed to mitigate the effect of the unpredictable nature of channel availability in cognitive radio networks with real-time traffic. It was shown that the proposed anticipated leasing strategy outperforms the dynamic leasing strategy in terms of maximum achieved Erlang capacity, without a significant penalty in terms of cost per rented resource per Erlang. Moreover, we found that anticipated and dynamic leasing strategies drastically reduce the cost per rented resource per Erlang relative to the permanent leasing strategy. Numerical results reveal that system performance strongly depends on both the mean secondary service time relative to the mean primary service time and the value of bandwidth requirement of both primary and secondary users. These sensitive issues have to be considered to adequately dimension and optimize cognitive radio networks with real-time traffic.

\section{Endnotes}

${ }^{1}$ Erlang capacity is defined as the maximum carried traffic that can be achieved by a given strategy in such a way that the different QoS requirements are met. 
${ }^{2}$ End-to-end QoS provisioning can be divided into two parts: QoS in the local (wireless) domain and QoS in the backbone domain. Since system impairments across the connection of the delay-sensitive application are cumulative, the required QoS at the local domain is determined by the QoS achieved at the backbone domain. After extraction of possible QoS values achieved at the backbone domain, the remaining QoS budget for the local domain is, in general, quite limited [34].

\section{Appendix}

It is important to consider the behavior of the system when the leasing and the reservation are both real numbers. For that motive, in this appendix, we take the Permanent Leasing Strategy, because it is the simplest one, and modified to illustrate the appropriate changes needed compared to the transition rates presented in Section 2.2. It is important to notice that for the dynamic leasing strategy, the mathematical analysis is similar. In this analysis, the following probabilities are considered: $P_{r}$ and $P_{R^{-}}$ defined as

$$
\begin{aligned}
& P_{r}=r-\lfloor r\rfloor \\
& P_{R}=R-\lfloor R\rfloor
\end{aligned}
$$

(A.1) represent the probability that a $\mathrm{SU}$ is using an extra reserved channel when there are $r$ reserved channels, while (A.2) represent the probability that a $\mathrm{SU}$ is using an extra-leased channel when there are $R$ leased channels.

The valid states space is given by:

$\Omega=\left\{(m, n) \mid 0 \leq \mathbf{b} \cdot \mathbf{e} \leq N+\lfloor R\rfloor+1 \cap m b_{m} \leq N \cap n b_{n} \leq N+\lfloor R\rfloor+1-\lfloor r\rfloor\right\}$

The arrival of a PU occurs with a rate:

$a_{m 1}(m, n)=\left\{\begin{aligned} \lambda_{m} & ; \quad m \geq 0 \cap \mathbf{b} \cdot \mathbf{e} \leq N+\lfloor R\rfloor-b_{m} \cap m b_{m} \leq N-b_{m} \\ P_{R} \lambda_{m} & ; \quad m \geq 0 \cap\left(\mathbf{b} \cdot \mathbf{e}>N+\lfloor R\rfloor-b_{m}\right) \cap(\mathbf{b} \cdot \mathbf{e} \leq N+\lfloor R\rfloor \\ 0 \quad & \left.+1-b_{m}\right) \cap m b_{m} \leq N-b_{m}\end{aligned}\right.$

Take notice that when the $R$ leased channels are being used, a new PU can be accepted with a probability $P_{R}$, guaranteeing that no $\mathrm{SU}$ is interrupted. On the other hand, the case when a PU arrives and $i$ SUs are forced to terminate happens with rate: $a_{m 2}(m, n)=\left\{\begin{aligned} &\left(1-P_{R}\right) \lambda_{m} \quad ; \quad m \geq 0 \cap\left(\mathbf{b} \cdot \mathbf{e}>N+\lfloor R\rfloor-b_{m}\right) \cap(\mathbf{b} \cdot \mathbf{e} \leq N \\ &\left.\quad+\lfloor R\rfloor+1-b_{m}\right) \cap m b_{m} \leq N-b_{m} \\ & \lambda_{m} ; \quad m \geq 0 \cap \mathbf{b} \cdot \mathbf{e}>N+\lfloor R\rfloor+1-b_{m} \cap m b_{m} \leq N-b_{m} \\ & 0 ; \quad \text { otherwise }\end{aligned}\right.$

The acceptance of a new SU session is bounded by the availability of leased and reserved channels. Because of that, it is possible to have multiple outcomes:

- A SU is admitted while there are $\lfloor R\rfloor$ channels rented and $\lfloor r\rfloor$ channels reserved; it will happen with a $\left(1-P_{R}\right)$ probability.

- A SU is admitted while there are $\lfloor R\rfloor$ channels rented and $\lfloor r\rfloor+1$ channels reserved; it will happen with a $\left(1-P_{R}\right)\left(1-P_{r}\right)$ probability.

- $\mathrm{A} S \mathrm{SU}$ is admitted while there are $\lfloor R\rfloor+1$ channels rented and $\lfloor r\rfloor$ channels reserved; it will happen with a $P_{R}$ probability.

- A SU is admitted while there are $\lfloor R\rfloor+1$ channels rented and $\lfloor r\rfloor+1$ channels reserved; it will happen with a $P_{R}\left(1-P_{r}\right)$ probability.

In summary the arrival of a SU happens with rate:

$a_{n}(m, n)=\left\{\begin{array}{rll}\lambda_{n} & ; \quad n \geq 0 \cap \mathbf{b} \cdot \mathbf{e} \leq N+\lfloor R\rfloor-\lfloor r\rfloor-1-b_{n} \\ \left(1-P_{r}+P_{r} P_{R}\right) \lambda_{n} & ; \quad n \geq 0 \cap \mathbf{b} \cdot \mathbf{e}=N+\lfloor R\rfloor-\lfloor r\rfloor-b_{n} \\ P_{R}\left(1-P_{r}\right) \lambda_{n} & ; \quad n \geq 0 \cap \mathbf{b} \cdot \mathbf{e}=N+\lfloor R\rfloor+1-\lfloor r\rfloor-b_{n} \\ 0 \quad ; & \text { otherwise }\end{array}\right.$

The rest of the rates are as follows:

the departure of a PU happens with rate:

$$
\begin{aligned}
& d_{m}(m, n) \\
& \quad= \begin{cases}m \mu_{m} ; & m>0 \cap m b_{m} \leq N \\
0 ; & \text { otherwise }\end{cases}
\end{aligned}
$$

and the departure of a $\mathrm{SU}$ is given with rate:

$$
\begin{aligned}
& d_{n}(m, n) \\
& \quad=\left\{\begin{array}{lll}
n \mu_{n} & ; \quad n>0 \cap n b_{n} \leq N+\lfloor R\rfloor+1-\lfloor r\rfloor \\
0 & ; \quad \text { otherwise }
\end{array}\right.
\end{aligned}
$$

Building on this, the blocking probabilities for PUs and SUs are given by: 


$$
P_{\mathrm{bm}}=\sum_{\left\{(m, n) \in \Omega \mid \mathrm{mb}_{m}=N\right\}} P(m, n)
$$

$$
\begin{aligned}
& P_{\text {bn }}=\sum_{\left\{(m, n) \in \Omega \mid\left(\mathbf{b} \cdot \mathbf{e}>N+\lfloor R\rfloor-\lfloor r\rfloor-1-b_{n}\right) \cap\left(\mathbf{b} \cdot \mathbf{e} \leq N+\lfloor R\rfloor-\lfloor r\rfloor-b_{n}\right)\right\}}\left(1-P_{R}\right) P_{r} P(m, n) \\
& +\sum_{\left\{(m, n) \in \Omega \mid\left(\mathbf{b} \cdot \mathbf{e}>N+\lfloor R\rfloor-\lfloor r\rfloor-b_{n}\right) n\left(\mathbf{b} \cdot \mathbf{e} \leq N+\lfloor R\rfloor+1-\lfloor r\rfloor-b_{n}\right)\right\}}\left(1-P_{R}+P_{r} P_{R}\right) P(m, n) \\
& +\sum_{\left\{(m, n) \in \Omega \mid\left(\mathbf{b} \cdot \mathbf{e}>N+\lfloor R\rfloor+1-\lfloor r\rfloor-b_{n}\right)\right\}} P(m, n)
\end{aligned}
$$

and the secondary forced termination probability can be computed as follows:

$$
\begin{aligned}
& P_{\mathrm{ftn}}=\frac{\left\{(m, n) \in \Omega \mid m \geq 0 \cap\left(\mathbf{b} \cdot \mathbf{e}>N+\lfloor R\rfloor-b_{m} \cap m b_{m} \leq N-b_{m}\right) \cap\left(\mathbf{b} \cdot \mathbf{e}<N+\lfloor R\rfloor+1-b_{m} \cap m b_{m} \leq N-b_{m}\right)\right\}}{\lambda_{n}\left(1-P b_{n}\right)}\lceil \\
& \sum_{b_{n}}\left\lceil\frac{\mathbf{b} \cdot \mathbf{e}-\left(N+R-b_{m}\right)}{b_{n}}\right\rceil \lambda_{m}\left(1-P_{R}\right) P(m, n) \\
& \left.+\frac{\left\{(m, n) \in \Omega \mid \mathbf{e}-\left(N+R+1-b_{m}\right)\right.}{b_{n}}\right\rceil \lambda_{m} P(m, n)
\end{aligned}
$$

\section{Funding}

PRODEP Contract No. 916039 provides us resources to acquire computing equipment for the numerical evaluation of the studied systems.

\section{Authors' contributions}

MAR-R developed mathematical analysis for the spectrum leasing strategies. He also obtained numerical results obtained to evaluate the performance of the system in terms of Erlang capacity. SLC- $L$ developed the teletraffic analysis and the optimization algorithm to maximize system Erlang capacity. FAC-P was involved in the development of the different mathematical models as well as identifying, analyzing, and studying the different factors that affect system performance. GH-V was involved in the development of the different mathematical models as well as identifying, analyzing, and studying the different factors that affect system performance. MER-A was involved in the mathematical analysis as well as in the numerical evaluation of the considered system. All authors read and approved the final manuscript.

\section{Authors' information}

M. A. R.-R. was born in Ocampo Tamaulipas, México, in 1989. He received the BSC degree from the Instituto Politécnico Nacional (IPN), México, in 2012 and the M.Sc degree from CINVESTAV-IPN in 2014 in Electrical Engineering. He is currently pursuing a Ph.D in Electrical Engineering at CINVESTAV-IPN. His research interest includes coordinated cognitive radio, teletraffic analysis for mobile networks, and resource management for wireless networks.

F. A. C.-P. was born in Mixquiahuala, Hidalgo, Mexico, in 1972. He received the B.Sc. degree from the Technological Institute and Superior Studies of Monterrey (ITESM), Mexico, in 1994 and the M.Sc. and Ph.D. degrees from CINVESTAV-IPN in 1997 and 2001, respectively, all in electrical engineering. In January 2001, he joined the Department of Electrical Engineering at
CINVESTAV-IPN as an Assistant Professor, where he earned early promotion to Associate Professor in April 2003. He has published over 100 papers in refereed professional journals and conferences.

His research interests span many areas including wireless networks, mobile computing, and mobile communications. His research interests include resource management, system level performance evaluation, teletraffic analysis, quality-of-service provisioning, call admission control, microcellular systems, cognitive radio systems, and wireless communication systems with link adaptation and/or smart antennas.

Dr. Cruz-Pérez is a Senior Member of the IEEE, a member of the IEICE, and professional member of the ACM. Since August 2001, he has been a member of the National System of Researchers in Mexico. Also, he is a regular member of the Mexican Academy of Sciences. Dr. Cruz-Pérez has been a member of the editorial board of the IEEE TRANSACTIONS ON VEHICULAR TECHNOLOGY, Wireless Communications and Mobile Computing, the Journal of Communications and Networks, the International Journal of Communication Systems, IEEE TRANSACTIONS ON WIRELESS COMMUNICATIONS, IEEE COMMUNICATIONS LETTERS, and Telecommunications Systems. Finally, he has served as a member of the technical program committee for approximately fifty international conferences. S. L. C.-L. was born in México City, D.F., Mexico, in 1982. She obtained the PhD degree in Electrical Engineering at the Center for Research and Advanced Studies of the National Polytechnic Institute (CINVESTAV-IPN) of Mexico in 2014. She is currently working at Autonomous Metropolitan University in Mexico City. Her research interests are in resource management, teletraffic analysis, quality-of-service provisioning, call admission control, and prioritized resource allocation in mobile wireless communication systems and cognitive radio systems.

G. H.-V. was born in México City, Mexico, in November 1968. He received the B.Sc. degree in electronics and communications engineering from the 
National Polytechnic Institute (IPN) in 1996 and the M.Sc. and Ph.D. degrees, both in electrical engineering, from CINVESTAV-IPN in 1997 and 2009, respectively, all in Mexico City, Mexico.

Since September 1996, he has been with the Department of Electronics Engineering at the Metropolitan Autonomous University campus Azcapotzalco (UAM-A) in Mexico City, Mexico, where he has been working as a Research Lecturer. His research interests include, but are not limited to, performance evaluation, teletraffic analysis, and resource management of wireless mobile communications networks.

M. E. R.-A. was born in México D.F., Mexico, in 1976. He received the BSc degree from Metropolitan Autonomous University (UAM), Mexico, in 1998 and the M.Sc. and Ph.D. degrees from CINVESTAV-IPN in 2000 and 2006, respectively, in Electrical Engineering. He is a professor at the National Polytechnic Institute, currently at the Center of Research in Computation (CIC-IPN), Mexico since 2002. He was a Postdoctoral Fellow at Dyonisos research project in INRIA (Institut National de Recherche en Informatique et en Automatique), Rennes, France from 2007 to 2010. His research interest includes random access protocols and data transmission in cellular networks, P2P networks, and wireless sensor networks.

\section{Competing interests}

The authors declare that they have no competing interests.

\section{Publisher's Note}

Springer Nature remains neutral with regard to jurisdictional claims in published maps and institutional affiliations.

\section{Author details}

${ }^{1}$ Electrical Engineering Department CINVESTAV-IPN, Mexico City, Mexico. 2Electronics Department UAM-A, Mexico City, Mexico. ${ }^{3}$ Communication Netwroks Lab. CIC-Instituto Politéccnico Nacional, Mexico City, Mexico.

Received: 27 June 2017 Accepted: 2 October 2018 Published online: 19 October 2018

\section{References}

1. Y.-C. Liang, K.-C. Chen, G.Y. Li, P. Mähönen, Cognitive radio networking and communications: an overview. IEEE Trans. Veh. Technol. 60(7), 3386-3407 (2011).

2. I.F. Akyildiz, W.Y. Lee, M.C. Vuran, S. Mohanty, NeXt generation/dynamic spectrum access/cognitive radio wireless networks: a survey. Computer Networks 50 (2006).

3. F. Wang, J. Huang, Y. Zhao, Delay sensitive communications over cognitive radio networks. IEEE Transactions on Wireless Communications 11(4), 14021411 (2012).

4. S. L. Castellanos-López, F.A. Cruz-Pérez, M.E. Rivero-Angeles, and G. Hernández-Valdez, "Joint connection level and packet level analysis of cognitive radio networks with VoIP traffic". IEEE Journal on Selected Areas in Communications: Cognitive Radio Series (2014).

5. S. L. Castellanos-Lopez, F. A. Cruz-Pérez, M. E. Rivero-Angeles and G. Hernandez-Valdez, "Erlang capacity in coordinated cognitive radio networks with stringent-delay applications," 2013 IEEE 24th Annual International Symposium on Personal, Indoor, and Mobile Radio Communications (PIMRC), (London, 2013). pp. 3166-3170.

6. A. Turhan, M Alanyali and D Starobinski, "Optimal admission control of secondary users in preemptive cognitive radio networks," 10th WiOpt, 1418, 138-144 (2012)..

7. L. Jiao, F.Y. Li, V. Pla, Modeling and performance analysis of channel assembling in multichannel cognitive radio networks with spectrum adaptation. IEEE Transactions on Vehicular Technology 61(6), 2686-2697 (2012).

8. J. Martinez-Bauset, A. Popescu, V. Pla, A. Popescu, in Proc. IEEE 8th EURO-NGI Conference In Next Generation Internet (NGI). Cognitive radio networks with elastic traffic (2012), pp. 17-24.

9. X Mao, H Ji, VCM Leung and M Li, "Performance enhancement for unlicensed users in coordinated cognitive radio networks via channel reservation" 2010 IEEE global telecommunications conference GLOBECOM 2010 (Miami, 2010), pp. 1-5.

10. S.K. Jayaweera, G. Vazquez-Vilar, C. Mosquera, Dynamic spectrum leasing: a new paradigm for spectrum sharing in cognitive radio networks. IEEE Transactions on Vehicular Technology 59(5), 2328-2339 (2010).
11. L. Duan, J. Huang, B. Shou, Investment and pricing with spectrum uncertainty: a cognitive operator's perspective. IEEE Transactions on Mobile Computing 10(11), 1590-1604 (2011).

12. L Duan, Gao L and J. Huang, "Contract-based cooperative spectrum sharing," in Proc. IEEE DySPAN'2011, 2011, pp. 399-407.

13. G. Zhang, K. Yang K, J. Song, and Y. Li, "Fair and efficient spectrum splitting for unlicensed secondary users in cooperative cognitive radio networks". Wireless Personal Communications 71(1), pp. 299-316. (2012).

14. X. Jiang, Y. Zhang, K.K. Wong, J.M. Kim, D.J. Edwards, Quality of serviceaware coordinated dynamic spectrum access: prioritized Markov model and call admission control. Wireless Communications and Mobile Computing 13(5), 510-524 (2011).

15. MM Buddhikot, and K Ryan, "Spectrum management in coordinated dynamic spectrum access based cellular networks," in Proc. DySPAN 2005. pp. 299-307 (2005).

16. J.M. Chapin, W.H. Lehr, Time-limited leases in radio systems. IEEE Commun. Mag. 45(6), 76-82 (2007)

17. P.K. Tang, Y.H. Chew, W.L. Yeow, L.C. Ong, Performance comparison of three spectrum admission control policies in coordinated dynamic spectrum sharing systems. IEEE Trans. Veh. Technol. 58(7), 3674-3683 (2009).

18. I.A. Balapuwaduge, F.Y. Li, A. Rajanna, M. Kaveh, Channel occupancy-based dynamic spectrum leasing in multichannel CRNs: strategies and performance evaluation. IEEE Transactions on Communications 64(3), 13131328 (2016)

19. Y. Ma, X. Yin, X. Yang, Q. Liu, Resource allocation for spectrum-leasing based CRN with delay-sensitive traffic. EURASIP J. Wirel. Commun. Netw. 2017(1), 108 (2017).

20. N. Vassileva, Y. Koucheryavy, Effect of AMC on fixed-rate traffic with hard delay constraints in mobile broadband systems. Wirel. Commun. Mob. Comput. 15(4), 666-684 (2015).

21. D. Pacheco-Paramo, V. Pla and J Martinez-Bauset, "Optimal admission control in cognitive radio networks," 2009 4th international conference on cognitive radio oriented wireless networks and communications (Hannover, 2009). pp. 1-7.

22. Maryam Hafeez and Elmirghani Jaafar, "Dynamic spectrum leasing for cognitive radio networks - modelling and analysis,' energy management in wireless cellular and ad-hoc networks. (Springer International Publishing, 2016). p. 217-245

23. R. Fan, W. Chen, H. Jiang, J. An, K. Yang, C. Xing, Dynamic spectrum leasing with two sellers. IEEE Transactions on Vehicular Technology 67(6), 48524866 (2018).

24. Show-Shiow Tzeng, and Hsu Yu-Ching "Channel reservation in cellular wireless networks with Spectrum leasing." 2010 Proceedings of 19th International Conference on Computer Communications and Networks (2010)..

25. A.S. Shafigh et al., Semi-cognitive radio networks: a novel dynamic spectrum sharing mechanism. IEEE Transactions on Cognitive Communications and Networking 3(1), 97-111 (2017).

26. MA Ramirez-Reyna, FA Cruz-Perez, ME Rivero-Angeles and G HernandezValdez, "Performance analysis of dynamic spectrum leasing strategies in overlay cognitive radio networks," 2014 IEEE 80th Vehicular Technology conference (VTC2014-fall), (Vancouver, 2014). p. 1-7.

27. M. A. Ramirez-Reyna, F. A. Cruz-Pérez, M. E. Rivero-Angeles and G. Hernandez-Valdez "Dynamic spectrum leasing strategies for coordinated cognitive radio networks with delay-tolerant traffic," 2014 IEEE 25th Annual International Symposium on Personal, Indoor, and Mobile Radio Communication (PIMRC), (Washington DC, 2014). p. 1200-1205..

28. S.L. Castellanos-López, F.A. Cruz-Pérez, M.E. Rivero-Ángeles, G. HernándezValdez, Performance analysis of coordinated cognitive radio networks under fixed-rate traffic with hard delay constraints. Journal of Communications and Networks 16(2), 130-139 (2014).

29. R. Ramjee, D. Towsley, R. Nagarajan, On optimal call admission control in cellular networks. Wireless Networks 3(1), 29-41 (1997).

30. S. Tang, B.L. Mark, Modeling and analysis of opportunistic spectrum sharing with unreliable spectrum sensing. IEEE Transactions on Wireless Communications 8(4), 1934-1943 (2009).

31. I. Suliman, J. Lehtomäki, T. Bräysy and K. Umebayashi, "Analysis of cognitive radio networks with imperfect sensing," 2009 IEEE 20th International Symposium on Personal, Indoor and Mobile Radio Communications, Tokyo, 2009, pp. 1616-1620.

32. X. Gelabert, O. Salient, J. Prez-Romero, R. Agust, Spectrum sharing in cognitive radio networks with imperfect sensing: a discrete-time Markov model. Computer Networks 54(14), 2519-2536 (2010). 
33. S. Tang, "A general model of opportunistic spectrum sharing with unreliable sensing". International Journal Communications Systems. (2012). https://doi. org/10.1002/dac.2340.

34. K. Kassev, Y. Mihov, A. Kalaydzhieva and B.Tsankov, "A new paradigm of CAC dimensioning for VolP traffic over wireless access networks," 2010 fourth international conference on digital society, (St. Maarten, 2010), pp. 54-59.

Submit your manuscript to a SpringerOpen ${ }^{\circ}$ journal and benefit from:

- Convenient online submission

- Rigorous peer review

- Open access: articles freely available online

High visibility within the field

- Retaining the copyright to your article

Submit your next manuscript at $\boldsymbol{\sim}$ springeropen.com 